\title{
Cauchy problems for thermoelastic plate equations with different damping mechanisms
}

\author{
Wenhui Chen
}

\begin{abstract}
In this paper we study Cauchy problem for thermoelastic plate equations with friction or structural damping in $\mathbb{R}^{n}, n \geq 1$, where the heat conduction is modeled by Fourier's law. We explain some qualitative properties of solutions influenced by different damping mechanisms. We show which damping in the model has a dominant influence on smoothing effect, energy estimates, $L^{p}-L^{q}$ estimates not necessary on the conjugate line, and on diffusion phenomena. Moreover, we derive asymptotic profiles of solutions in a framework of weighted $L^{1}$ data. In particular, sharp decay estimates for lower bound and upper bound of solutions in the $\dot{H}^{s}$ norm $(s \geq 0)$ are shown.
\end{abstract}

Mathematics Subject Classification (2010). Primary 35Q99; Secondary 35B40, 74F05.

Keywords. Thermoelastic plate equations, Fourier's law, friction, structural damping, diffusion phenomena, asymptotic profiles.

\section{Introduction}

In recent years, thermoelastic plate equations have attracted a lot of attention. The Cauchy problem for linear thermoelastic plate equations are modeled by

$$
\begin{cases}u_{t t}+\Delta^{2} u+\Delta \theta=0, & t>0, x \in \mathbb{R}^{n}, \\ \theta_{t}-\Delta \theta-\Delta u_{t}=0, & t>0, x \in \mathbb{R}^{n}, \\ \left(u, u_{t}, \theta\right)(0, x)=\left(u_{0}, u_{1}, \theta_{0}\right)(x), & x \in \mathbb{R}^{n},\end{cases}
$$

where the unknowns $u=u(t, x)$ and $\theta=\theta(t, x)$ denote the elongation of a plate and the temperature difference to the equilibrium state, respectively. The recent papers $[34,32]$ investigated $L^{2}$-decay estimates of solutions to (1.1) by using energy methods in the Fourier space. Simultaneously, [32] proved the sharpness of the derived decay estimates by applying asymptotic expansions of eigenvalues. Other studies on thermoelastic plate equations can be found in the literature. We refer to [1, 19, 20, $21,22,23,24,26,27]$ for the initial boundary value problem in bounded domains, $[5,6,7,26,27]$ for the Cauchy problem or in general exterior domains.

In the real world and applications, due to some kinds of resistance in the elongation of a plate, we always model a plate equation with damping terms, for instance, plate equations with structural damping in $[12,17]$. When the thermal dissipation modeled by Fourier's law and the dissipation for the elongation of a plate appear at the same time, we model thermoelastic plate equations with an additional damping in the equation for $u$, for example, the thermoelastic plate equations with friction $u_{t}$ presented in [36], with structural damping $-\Delta u_{t}$ presented in $[9,11]$, with Kelvin-Voigt type damping or viscoelastic damping $\Delta^{2} u_{t}$ presented in [38]. For this reason, we consider thermoelastic plate equations with different damping mechanisms in present paper. 
In this paper we are concerned with the following Cauchy problem for thermoelastic plate equations in $\mathbb{R}^{n}, n \geq 1$, where the heat conduction is modeled by Fourier's law:

$$
\begin{cases}u_{t t}+\Delta^{2} u+\Delta \theta+(-\Delta)^{\sigma} u_{t}=0, & t>0, x \in \mathbb{R}^{n} \\ \theta_{t}-\Delta \theta-\Delta u_{t}=0, & t>0, x \in \mathbb{R}^{n} \\ \left(u, u_{t}, \theta\right)(0, x)=\left(u_{0}, u_{1}, \theta_{0}\right)(x), & x \in \mathbb{R}^{n}\end{cases}
$$

where $\sigma \in[0,2]$. To be more specific, $\sigma=0$ stands for the system with friction or external damping, $\sigma \in(0,2]$ stands for the system with structural damping, especially, $\sigma=2$ stands for the system with Kelvin-Voigt type damping. The example of the model of thermoealstic plate equations with friction or structural damping (1.2) is a special case of $\alpha-\beta-\gamma$ systems, which have been introduced in [10],

$$
\left\{\begin{array}{l}
u_{t t}+\mathscr{A} u-\mathscr{A}^{\beta} \theta+\mathscr{A}^{\gamma} u_{t}=0 \\
\theta_{t}+\mathscr{A}^{\alpha} \theta+\mathscr{A}^{\beta} u_{t}=0
\end{array}\right.
$$

when we choose

$$
\mathscr{A}=(-\Delta)^{2} \text { and } \alpha=\beta=\frac{1}{2}, \quad[0,1] \ni \gamma=\frac{1}{2} \sigma .
$$

Nevertheless, the influence of an additional damping in the equation for $u$ in thermoelastic plate equations on some qualitative properties of solutions as $L^{p}-L^{q}$ estimates, diffusion phenomena, asymptotic profiles of solutions is still open.

Our main purpose of this paper is to study different qualitative properties of solutions to the thermoelastic plate equations with different damping mechanisms. More specifically, we are interested in the following properties of solutions to (1.2):

(1) smoothing effect and $L^{2}$ well-posedness;

(2) energy estimates with different assumptions on initial data;

(3) $L^{p}-L^{q}$ estimates not necessary on the conjugate line;

(4) diffusion phenomena;

(5) asymptotic profiles of solutions in a framework of weighted $L^{1}$ data.

Then, due to the fact that different kinds of damping (friction, structural damping, thermal damping) have different influence on the model, we will analyze the dominant influence from the damping to different qualitative properties of solutions. In other words, there exists a competition between "friction or structural damping" and "thermal damping generated by Fourier's law".

In order to study the above qualitative properties of solutions, especially, $L^{p}-L^{p}$ estimates for $1 \leq p \leq \infty$, diffusion phenomena and asymptotic profiles of solutions, we need to derive representations of solutions instead of using pointwise estimates in the Fourier space. However, because the fractional power operator $(-\Delta)^{\sigma}$ acts on $u_{t}$ in the damping term, the method of asymptotic expansions of eigenprojections (c.f. $[13,2])$ seems to be difficult to applied. Moreover, the method of asymptotic expansions of eigenvalues (c.f. [32, 13]) cannot be used directly to prove the sharpness for the derived estimates of solutions. To overcome these difficulties, we may derive representations of solutions by applying methods of WKB analysis. The main tool is the application of a multi-step diagonalization procedure, which was mainly proposed in $[18,31]$.

In the study of diffusion phenomena of solutions to (1.2), we may observe the corresponding reference system to (1.2), which is consisted of different evolution equations, e.g., heat equation, fractional heat equation, Schrödinger equation, fourth-order parabolic equation. The equations of such reference system are determined by the value of $\sigma$ in the damping term $(-\Delta)^{\sigma} u_{t}$. It will provide some opportunities for us to understand the model (1.2) in a more precise way. 
For asymptotic profiles of solutions in a framework of weighted $L^{1}$ data in Section 6 , by introducing

$$
\begin{aligned}
U(t, x) & :=\left(u_{t}+|D|^{2} u, u_{t}-|D|^{2} u, \theta\right)^{\mathrm{T}}(t, x), \\
U_{0}(x) & :=\left(u_{1}+|D|^{2} u_{0}, u_{1}-|D|^{2} u_{0}, \theta_{0}\right)^{\mathrm{T}}(x), \\
P_{U_{0}} & :=\int_{\mathbb{R}^{n}} U_{0}(x) d x \quad \text { with } \quad\left|P_{U_{0}}\right| \neq 0,
\end{aligned}
$$

we will prove the following estimates for $t \gg 1$ :

$$
t^{-\frac{n+2 s}{4 \max \{2-\sigma ; 1\}}}\left|P_{U_{0}}\right| \lesssim\|U(t, \cdot)\|_{\dot{H}^{s}\left(\mathbb{R}^{n}\right)} \lesssim t^{-\frac{n+2 s}{4 \max \{2-\sigma ; 1\}}}\left\|U_{0}\right\|_{H^{s}\left(\mathbb{R}^{n}\right) \cap L^{1,1}\left(\mathbb{R}^{n}\right)},
$$

with $s \geq 0, \sigma \in[0,2]$. It immediately leads to sharp decay rate of the estimates for the $\dot{H}^{s}$ norm of solutions. To the best of the author's knowledge, sharp estimates for lower bound of solutions for dissipative elastic systems are unknown, although the estimates for upper bound of solutions in the $L^{2}$ norm have been extensively discussed in several kinds of elastic systems, see $[29,3,2]$ for dissipative elastic waves, [18, 31, 37, 40, 41] for thermoelastic systems. We remark that our method can probably applied to some other systems in elastic material (see Remark 7.1), too. Furthermore, due to the double damping, including friction or structural damping and thermal damping generated by Fourier's law, it is interesting to investigate which damping will give stronger effects on the asymptotic profiles of solutions.

The paper is organized as follows. In Section 2, we prepare representations of solutions to (1.2) by WKB analysis. In Section 3, by using these representations of solutions we study smoothing effect of solutions and $L^{2}$ well-posedness of the Cauchy problem (1.2). In Section 4, we derive some estimates of solutions, including energy estimates with initial data taking from $H^{s}\left(\mathbb{R}^{n}\right) \cap L^{m}\left(\mathbb{R}^{n}\right)$ with $s \geq 0$, $m \in[1,2]$, and $L^{p}-L^{q}$ estimates not necessary on the conjugate line. In Section 5 , diffusion phenomena for linear thermoelastic plate equations with friction or structural damping are investigated. In Section 6 , we derive long-time asymptotic profiles of solutions in a framework of weighted $L^{1}$ data. Finally, in Section 7 some concluding remarks complete the paper.

Notation. We now give some notations to be used in this paper. We denote the identity matrix of dimension $k \times k$ by $I_{k} . f \lesssim g$ means that there exists a positive constant $C$ such that $f \leq C g$. Moreover, $H_{p}^{s}\left(\mathbb{R}^{n}\right)$ and $\dot{H}_{p}^{s}\left(\mathbb{R}^{n}\right)$ with $s \geq 0$ and $1 \leq p<\infty$, denote Bessel and Riesz potential spaces based on $L^{p}\left(\mathbb{R}^{n}\right)$, respectively. Here $\langle D\rangle^{s}$ and $|D|^{s}$ stand for the pseudo-differential operators with symbols $\langle\xi\rangle^{s}$ and $|\xi|^{s}$, respectively.

Let us define the Gevrey spaces $\Gamma^{\kappa}\left(\mathbb{R}^{n}\right)$ for $\kappa \in[1, \infty)$ by (c.f. [33])

$$
\Gamma^{\kappa}\left(\mathbb{R}^{n}\right):=\left\{f \in L^{2}\left(\mathbb{R}^{n}\right): \text { there exists a constant } c \text { such that } \exp \left(c\langle\xi\rangle^{\frac{1}{\kappa}}\right) \hat{f} \in L^{2}\left(\mathbb{R}^{n}\right)\right\} .
$$

Let us define the weighted $L^{1}$ spaces $L^{1, \delta}\left(\mathbb{R}^{n}\right)$ for $\delta \in[0, \infty)$ by

$$
L^{1, \delta}\left(\mathbb{R}^{n}\right):=\left\{f \in L^{1}\left(\mathbb{R}^{n}\right):\|f\|_{L^{1, \delta}\left(\mathbb{R}^{n}\right)}:=\int_{\mathbb{R}^{n}}(1+|x|)^{\delta}|f(x)| d x\right\} .
$$

Particularly, we notice that $L^{1,0}\left(\mathbb{R}^{n}\right)=L^{1}\left(\mathbb{R}^{n}\right)$.

\section{Asymptotic behavior of solutions}

First of all, we apply the partial Fourier transformation with respect to spatial variables to (1.2) to get the following second-order ordinary differential system:

$$
\begin{cases}\hat{u}_{t t}+|\xi|^{4} \hat{u}-|\xi|^{2} \hat{\theta}+|\xi|^{2 \sigma} \hat{u}_{t}=0, & t>0, \xi \in \mathbb{R}^{n} \\ \hat{\theta}_{t}+|\xi|^{2} \hat{\theta}+|\xi|^{2} \hat{u}_{t}=0, & t>0, \xi \in \mathbb{R}^{n} \\ \left(\hat{u}, \hat{u}_{t}, \hat{\theta}\right)(0, \xi)=\left(\hat{u}_{0}, \hat{u}_{1}, \hat{\theta}_{0}\right)(\xi), & \xi \in \mathbb{R}^{n} .\end{cases}
$$


Introducing a new ansatz $w^{(0)}=w^{(0)}(t, \xi)$ by

$$
w^{(0)}:=\left(\hat{u}_{t}+|\xi|^{2} \hat{u}, \hat{u}_{t}-|\xi|^{2} \hat{u}, \hat{\theta}\right)^{\mathrm{T}},
$$

we obtain the first-order system as follows:

$$
\begin{cases}w_{t}^{(0)}+\left(|\xi|^{2} A_{0}+|\xi|^{2 \sigma} A_{1}\right) w^{(0)}=0, & t>0, \xi \in \mathbb{R}^{n} \\ w^{(0)}(0, \xi)=w_{0}^{(0)}(\xi), & \xi \in \mathbb{R}^{n}\end{cases}
$$

where $w_{0}^{(0)}=w_{0}^{(0)}(\xi)$ is defined by

$$
w_{0}^{(0)}:=\left(\hat{u}_{1}+|\xi|^{2} \hat{u}_{0}, \hat{u}_{1}-|\xi|^{2} \hat{u}_{0}, \hat{\theta}_{0}\right)^{\mathrm{T}},
$$

and the coefficient matrices are given by

$$
A_{0}=\frac{1}{2}\left(\begin{array}{ccc}
0 & -2 & -2 \\
2 & 0 & -2 \\
1 & 1 & 2
\end{array}\right) \text { and } A_{1}=\frac{1}{2}\left(\begin{array}{ccc}
1 & 1 & 0 \\
1 & 1 & 0 \\
0 & 0 & 0
\end{array}\right) .
$$

Additionally, we denote the matrix

$$
A(|\xi| ; \sigma):=|\xi|^{2} A_{0}+|\xi|^{2 \sigma} A_{1},
$$

and

$$
\begin{aligned}
U(t, x) & :=\left(u_{t}+|D|^{2} u, u_{t}-|D|^{2} u, \theta\right)^{\mathrm{T}}(t, x), \\
U_{0}(x) & :=\left(u_{1}+|D|^{2} u_{0}, u_{1}-|D|^{2} u_{0}, \theta_{0}\right)^{\mathrm{T}}(x) .
\end{aligned}
$$

It is clear that $\mathscr{F}_{x \rightarrow \xi}(U(t, x))=w^{(0)}(t, \xi)$ and $\mathscr{F}\left(U_{0}(x)\right)=w_{0}^{(0)}(\xi)$.

\subsection{Diagonalization schemes}

In the beginning, we divide the phase space into three regions

$$
\begin{aligned}
Z_{\text {int }}(\varepsilon) & =\left\{\xi \in \mathbb{R}^{n}:|\xi| \leq \varepsilon \ll 1\right\}, \\
Z_{\text {mid }}(\varepsilon, N) & =\left\{\xi \in \mathbb{R}^{n}: \varepsilon \leq|\xi| \leq N\right\}, \\
Z_{\text {ext }}(N) & =\left\{\xi \in \mathbb{R}^{n}:|\xi| \geq N \gg 1\right\},
\end{aligned}
$$

for small, bounded and large frequencies. Later, we will diagonalize the principle part of the first-order system (2.4) in each region. Furthermore, let us define $\chi_{\text {int }}(\xi), \chi_{\text {mid }}(\xi), \chi_{\text {ext }}(\xi) \in \mathscr{C}^{\infty}\left(\mathbb{R}^{n}\right)$ having their supports in $Z_{\text {int }}(\varepsilon), Z_{\text {mid }}(\varepsilon, N)$ and $Z_{\text {ext }}(N)$, respectively, so that $\chi_{\text {mid }}(\xi)=1-\chi_{\text {int }}(\xi)-\chi_{\text {ext }}(\xi)$.

To understand the influence of the parameter $\xi$ on the asymptotic behavior of solutions, we now distinguish between the next four cases.

- Case 2.1: $\sigma \in[0,1)$ with $\xi \in Z_{\text {int }}(\varepsilon)$ or $\sigma \in(1,2]$ with $\xi \in Z_{\text {ext }}(N)$;

- Case 2.2: $\sigma \in[0,1)$ with $\xi \in Z_{\text {ext }}(N)$ or $\sigma \in(1,2]$ with $\xi \in Z_{\text {int }}(\varepsilon)$;

- Case 2.3: $\sigma=1$ for all frequencies;

- Case 2.4: $\sigma \neq 1$ with $\xi \in Z_{\text {mid }}(\varepsilon, N)$.

For frequencies in the small zone or the large zone, i.e., Cases 2.1 and 2.2, the diagonalization procedure is available. This procedure, which is developed $[18,31,39]$, allows us to derive representations of solutions. For Case 2.3 , the matrix $A(|\xi| ; 1)$ may be understood as no perturbed linear operator for all frequencies due to $A(|\xi| ; 1)=|\xi|^{2}\left(A_{0}+A_{1}\right)$. For this reason, we only need to calculate the eigenvalues of the matrix $A(|\xi| ; 1)$ directly in Case 2.3. For frequencies in the bounded zone and $\sigma \neq 1$, i.e., Case 2.4 , we construct a contradiction to prove that the real parts of the characteristic roots have a fixed sign. 
Lemma 2.1 (Treatment for Case 2.1). When $\sigma \in[0,1)$ with $\xi \in Z_{\text {int }}(\varepsilon)$, or $\sigma \in(1,2]$ with $\xi \in Z_{\text {ext }}(N)$, after $\ell$ steps of diagonalization procedure the starting system (2.3) is transformed to

$$
\begin{cases}w_{t}^{(\ell)}-\left(\Lambda_{0}+\cdots+\Lambda_{\ell}+R_{\ell+1}\right) w^{(\ell)}=0, & t>0, \xi \in \mathbb{R}^{n}, \\ w^{(\ell)}(0, \xi)=w_{0}^{(\ell)}(\xi), & \xi \in \mathbb{R}^{n},\end{cases}
$$

with the diagonalized matrices $\Lambda_{1}, \ldots, \Lambda_{\ell}$ and the remainder $R_{\ell+1}$. The asymptotic behavior of these matrices can be described as follows:

$$
\Lambda_{0}=\mathcal{O}\left(|\xi|^{2 \sigma}\right), \quad \Lambda_{j}=\mathcal{O}\left(|\xi|^{2(1-\sigma)(j-1)+2}\right), \quad R_{\ell+1}=\mathcal{O}\left(|\xi|^{2(1-\sigma) \ell+2}\right) .
$$

Moreover, the characteristic roots $\lambda_{\ell, j}=\lambda_{\ell, j}(|\xi|)$ with $j=1,2,3$, having the following asymptotic behavior:

$$
\lambda_{\ell, 1}=|\xi|^{4-2 \sigma}, \quad \lambda_{\ell, 2}=|\xi|^{2}+|\xi|^{4-2 \sigma}, \quad \lambda_{\ell, 3}=|\xi|^{2 \sigma}-2|\xi|^{4-2 \sigma},
$$

modulo $O\left(|\xi|^{6-4 \sigma}\right)$.

Proof. To start the diagonalization procedure, the matrix $|\xi|^{2 \sigma} A_{1}$ has a dominant influence in comparison with the matrix $|\xi|^{2} A_{0}$ in Case 2.1. As the consequence, we should diagonalize $|\xi|^{2 \sigma} A_{1}$ in the first place. With the aid of variable change

$$
w^{(1)}:=T_{0}^{-1} w^{(0)}:=\left(\begin{array}{ccc}
-1 & 0 & 1 \\
1 & 0 & 1 \\
0 & 1 & 0
\end{array}\right)^{-1} w^{(0)},
$$

we derive

$$
w_{t}^{(1)}+\left(\Lambda_{0}+R_{1}\right) w^{(1)}=0
$$

where

$$
\Lambda_{0}=\operatorname{diag}\left(0,0,|\xi|^{2 \sigma}\right)=\circlearrowleft\left(|\xi|^{2 \sigma}\right)
$$

and $R_{1}=|\xi|^{2} A_{0}^{(1)}=\mathcal{O}\left(|\xi|^{2}\right)$ with

$$
A_{0}^{(1)}=T_{0}^{-1} A_{0} T_{0}=\left(\begin{array}{ccc}
0 & 0 & 1 \\
0 & 1 & 1 \\
-1 & -1 & 0
\end{array}\right)=\mathcal{O}(1) .
$$

Next, we define

$$
w^{(2)}:=T_{1}^{-1} w^{(1)}
$$

with $T_{1}:=I_{3}+N_{1}(|\xi|)$, where

$$
N_{1}(|\xi|):=|\xi|^{2-2 \sigma}\left(\begin{array}{ccc}
0 & 0 & 1 \\
0 & 0 & 1 \\
1 & 1 & 0
\end{array}\right)=\sigma\left(|\xi|^{2-2 \sigma}\right) .
$$

Thus, we have

$$
w_{t}^{(2)}+\left(\Lambda_{0}+T_{1}^{-1}\left(|\xi|^{2} A_{0}^{(1)}-\left[N_{1}(|\xi|), \Lambda_{0}\right]\right)+|\xi|^{2} T_{1}^{-1} A_{0}^{(1)} N_{1}(|\xi|)\right) w^{(2)}=0,
$$

where we use

$$
\left[N_{1}(|\xi|), \Lambda_{0}\right]:=N_{1}(|\xi|) \Lambda_{0}-\Lambda_{0} N_{1}(|\xi|)=|\xi|^{2}\left(\begin{array}{ccc}
0 & 0 & 1 \\
0 & 0 & 1 \\
-1 & -1 & 0
\end{array}\right) .
$$

By the relation $T_{1}^{-1}=I_{3}-T_{1}^{-1} N_{1}(|\xi|)$, we transform (2.7) to the following first-order system:

$$
w_{t}^{(2)}+\left(\Lambda_{0}+\Lambda_{1}+R_{2}\right) w^{(2)}=0
$$


where

$$
\Lambda_{1}:=\operatorname{diag}\left(0,|\xi|^{2}, 0\right)=\mathcal{O}\left(|\xi|^{2}\right)
$$

and $R_{2}=A_{0}^{(2)}-T_{1}^{-1} N_{1}(|\xi|) A_{0}^{(2)}=\odot\left(|\xi|^{4-2 \sigma}\right)$ with

$$
A_{0}^{(2)}=-N_{1}(|\xi|) \Lambda_{1}+|\xi|^{2} A_{0}^{(1)} N_{1}(|\xi|)=|\xi|^{4-2 \sigma}\left(\begin{array}{ccc}
1 & 1 & 0 \\
1 & 1 & 1 \\
0 & -1 & -2
\end{array}\right)=\mathcal{O}\left(|\xi|^{4-2 \sigma}\right) .
$$

By the similar procedure, we introduce

$$
w^{(3)}:=T_{2}^{-1} T_{1 \frac{1}{2}}^{-1} w^{(2)}
$$

with $T_{1 \frac{1}{2}}:=I_{3}+N_{1 \frac{1}{2}}(|\xi|)$ and $T_{2}:=I_{3}+N_{2}(|\xi|)$, where

$$
\begin{aligned}
& N_{1 \frac{1}{2}}(|\xi|):=|\xi|^{4-4 \sigma}\left(\begin{array}{ccc}
0 & 0 & 0 \\
0 & 0 & 1 \\
0 & 1 & 0
\end{array}\right)=\mathcal{O}\left(|\xi|^{4-4 \sigma}\right), \\
& N_{2}(|\xi|):=|\xi|^{2-2 \sigma}\left(\begin{array}{ccc}
0 & 1 & 0 \\
-1 & 0 & 0 \\
0 & 0 & 0
\end{array}\right)=O\left(|\xi|^{2-2 \sigma}\right) .
\end{aligned}
$$

So, we derive the following system:

$$
w_{t}^{(3)}+\left(\Lambda_{0}+\Lambda_{1}+\Lambda_{2}+R_{3}\right) w^{(3)}=0,
$$

where

$$
\Lambda_{2}=\operatorname{diag}\left(|\xi|^{4-2 \sigma},|\xi|^{4-2 \sigma},-2|\xi|^{4-2 \sigma}\right)=\mathcal{O}\left(|\xi|^{4-2 \sigma}\right)
$$

and $R_{3}=\sigma\left(|\xi|^{6-4 \sigma}\right)$. Notice that the matrices $T_{\sigma, \text { int }}$ and $T_{\sigma \text {,ext }}$, respectively, are defined by

$$
\begin{aligned}
& T_{\sigma, \text { int }}:=T_{0} T_{1} T_{1 \frac{1}{2}} T_{2} \quad \text { if } \quad \sigma \in[0,1), \\
& T_{\sigma, \text { ext }}:=T_{0} T_{1} T_{1 \frac{1}{2}} T_{2} \quad \text { if } \quad \sigma \in(1,2] .
\end{aligned}
$$

Then, we carry out further steps of diagonalization proposed in [30, 39] to complete the proof.

Lemma 2.2 (Treatment for Case 2.2). When $\sigma \in[0,1)$ with $\xi \in Z_{\text {ext }}(N)$, or $\sigma \in(1,2]$ with $\xi \in Z_{\text {int }}(\varepsilon)$, after $\ell$ steps of diagonalization procedure the starting system (2.3) is transformed to

$$
\begin{cases}w_{t}^{(\ell)}-\left(\Lambda_{0}+\cdots+\Lambda_{\ell}+R_{\ell+1}\right) w^{(\ell)}=0, & t>0, \xi \in \mathbb{R}^{n} \\ w^{(\ell)}(0, \xi)=w_{0}^{(\ell)}(\xi), & \xi \in \mathbb{R}^{n}\end{cases}
$$

with the diagonalized matrices $\Lambda_{1}, \ldots, \Lambda_{\ell}$ and the remainder $R_{\ell+1}$. The asymptotic behavior of these matrices can be described as follows:

$$
\Lambda_{0}=\mathcal{O}\left(|\xi|^{2}\right), \quad \Lambda_{j}=\mathcal{O}\left(|\xi|^{2(\sigma-1)(j-1)+2 \sigma}\right), \quad R_{\ell+1}=\mathcal{O}\left(|\xi|^{2(\sigma-1) \ell+2 \sigma}\right) .
$$

Moreover, the characteristic roots $\lambda_{\ell, j}=\lambda_{\ell, j}(|\xi|)$ with $j=1,2,3$, having the following asymptotic behavior:

$$
\lambda_{\ell, 1}=y_{1}|\xi|^{2}, \quad \lambda_{\ell, 2}=y_{2}|\xi|^{2}, \quad \lambda_{\ell, 3}=y_{3}|\xi|^{2},
$$

modulo $\mathcal{O}\left(|\xi|^{2 \sigma}\right)$, where the constants $y_{j}$ for $j=1,2,3$ have been shown in (2.9) later.

Proof. In this part, the matrix $|\xi|^{2} A_{0}$ has a dominant influence in comparison with the matrix $|\xi|^{2 \sigma} A_{1}$. Thus, we should diagonalize $|\xi|^{2} A_{0}$ firstly. After applying the substitution

$$
w^{(1)}:=T_{0}^{-1} w^{(0)},
$$

we arrive at the system

$$
w_{t}^{(1)}+\left(\Lambda_{0}+R_{1}\right) w^{(1)}=0,
$$


with the diagonal matrix

$$
\Lambda_{0}=|\xi|^{2} T_{0}^{-1} A_{0} T_{0}=\operatorname{diag}\left(y_{1}|\xi|^{2}, y_{2}|\xi|^{2}, y_{3}|\xi|^{2}\right)=\mathcal{O}\left(|\xi|^{2}\right)
$$

and the remainder $R_{1}=|\xi|^{2 \sigma} T_{0}^{-1} A_{1} T_{0}=\mathcal{O}\left(|\xi|^{2 \sigma}\right)$. In the above, the value of $y_{j}$ for $j=1,2,3$ are the solutions to the cubic equation

$$
y^{3}-y^{2}+2 y-1=0 .
$$

Then, from direct calculations the value $y_{j}$ for $j=1,2,3$, are given by

$$
y_{1}=\frac{1}{3}\left(1+z_{1}\right), \quad y_{2}=\frac{1}{3}\left(1-\frac{1}{2} z_{1}+\frac{\sqrt{3}}{2} i z_{2}\right), \quad y_{3}=\frac{1}{3}\left(1-\frac{1}{2} z_{1}-\frac{\sqrt{3}}{2} i z_{2}\right),
$$

where

$$
z_{1}=\sqrt[3]{\frac{1}{2}(3 \sqrt{69}+11)}-\sqrt[3]{\frac{1}{2}(3 \sqrt{69}-11)}, \quad z_{2}=\sqrt[3]{\frac{1}{2}(3 \sqrt{69}+11)}+\sqrt[3]{\frac{1}{2}(3 \sqrt{69}-11)} .
$$

Note that $y_{1} \neq y_{2} \neq y_{3}$ and the real part of $y_{j}$ are positive for all $j=1,2,3$. We now denote the matrices $T_{\sigma, \text { int }}$ and $T_{\sigma, \text { ext }}$, respectively, by

$$
\begin{aligned}
& T_{\sigma, \text { int }}:=T_{0} \text { if } \quad \sigma \in(1,2], \\
& T_{\sigma, \text { ext }}:=T_{0} \text { if } \quad \sigma \in[0,1) .
\end{aligned}
$$

Finally, one may apply further steps of diagonalization proposed in [30, 39] to complete the proof.

Lemma 2.3 (Treatment for Case 2.3). When $\sigma=1$ with $\xi \in \mathbb{R}^{n}$, the starting system (2.3) can be transformed to

$$
\begin{cases}w_{t}^{(1)}-\Lambda_{0} w^{(1)}=0, & t>0, \xi \in \mathbb{R}^{n}, \\ w^{(1)}(0, \xi)=w_{0}^{(1)}(\xi), & \xi \in \mathbb{R}^{n},\end{cases}
$$

with the diagonalize matrix $\Lambda_{0}=\operatorname{diag}\left(y_{4}|\xi|^{2}, y_{5}|\xi|^{2}, y_{6}|\xi|^{2}\right)$, where the constants $y_{j}$ for $j=4,5,6$ have been shown in (2.11) later.

Proof. Here the matrices $|\xi|^{2} A_{0}$ and $|\xi|^{2 \sigma} A_{1}$ with $\sigma=1$ have the same influence on the principle part. For this reason, the following system comes:

$$
w_{t}^{(0)}+A(|\xi| ; 1) w^{(0)}=0 .
$$

From direct calculation, we get

$$
\begin{aligned}
0=\operatorname{det}\left(A(|\xi| ; 1)-\lambda I_{3}\right) & =\left|\begin{array}{ccc}
\frac{1}{2}|\xi|^{2}-\lambda & \frac{1}{2}|\xi|^{2}-|\xi|^{2} & -|\xi|^{2} \\
\frac{1}{2}|\xi|^{2}+|\xi|^{2} & \frac{1}{2}|\xi|^{2}-\lambda & -|\xi|^{2} \\
\frac{1}{2}|\xi|^{2} & \frac{1}{2}|\xi|^{2} & |\xi|^{2}-\lambda
\end{array}\right| \\
& =-\lambda^{3}+2|\xi|^{2} \lambda^{2}-3|\xi|^{4} \lambda+|\xi|^{6} \\
& =-|\xi|^{6}\left(\left(\frac{\lambda}{|\xi|^{2}}\right)^{3}-2\left(\frac{\lambda}{|\xi|^{2}}\right)^{2}+3\left(\frac{\lambda}{|\xi|^{2}}\right)-1\right)
\end{aligned}
$$

In other words, we only need to study the solution to the cubic equation

$$
y^{3}-2 y^{2}+3 y-1=0 .
$$

By a simple calculation, we find the solution to above cubic equation given by

$$
y_{4}=z_{3}-\frac{5}{9 z_{3}}+\frac{2}{3}, \quad y_{5}=z_{4}-\frac{5}{9 z_{4}}+\frac{2}{3}, \quad y_{6}=z_{5}-\frac{5}{9 z_{5}}+\frac{2}{3},
$$

where

$$
z_{3}=\frac{1}{3} \sqrt[3]{-\frac{11}{2}+\frac{3}{2} \sqrt{69}}, \quad z_{4}=\left(-\frac{1}{2}+\frac{\sqrt{3}}{2} i\right) z_{3}, \quad z_{5}=\left(-\frac{1}{2}-\frac{\sqrt{3}}{2} i\right) z_{3}
$$


Note that $y_{4} \neq y_{5} \neq y_{6}$ with Re $y_{j}>0$ for $j=4,5,6$.

By introducing a new ansatz

$$
w^{(1)}:=T_{1,0}^{-1} w^{(0)},
$$

we obtain the following system:

$$
w_{t}^{(1)}+\Lambda_{0} w^{(1)}=0
$$

with the diagonal matrix

$$
\Lambda_{0}=T_{1,0}^{-1} A(|\xi| ; 1) T_{1,0}=\operatorname{diag}\left(y_{4}|\xi|^{2}, y_{5}|\xi|^{2}, y_{6}|\xi|^{2}\right)=\mathcal{O}\left(|\xi|^{2}\right) .
$$

Then, the proof of this lemma is completed.

Lastly, we derive an exponential decay result for frequencies in the bounded zone $Z_{\text {mid }}(\varepsilon, N)$ to guarantee the stability of solutions to (2.4) for $\sigma \in[0,1) \cup(1,2]$.

Lemma 2.4 (Treatment for Case 2.4). The solution $w^{(0)}=w^{(0)}(t, \xi)$ to the Cauchy problem (2.4) with $\sigma \in[0,1) \cup(1,2]$ satisfies

$$
\left|w^{(0)}(t, \xi)\right| \lesssim e^{-c t}\left|w_{0}^{(0)}(\xi)\right|
$$

for $\xi \in Z_{\text {mid }}(\varepsilon, N)$, where $c$ is a positive constant.

Proof. The following considerations help us obtain a priori estimate for the characteristic roots for frequencies in the bounded zone $Z_{\text {mid }}(\varepsilon, N)$. We assume that there is a purely imaginary eigenvalue $\lambda=i a$ with $a \in \mathbb{R} \backslash\{0\}$ of the coefficient matrix $A(|\xi| ; \sigma)$ for $\xi \neq 0$. The eigenvalue $\lambda$ satisfies the following cubic equation:

$$
\begin{aligned}
0=\operatorname{det}\left(A(|\xi| ; \sigma)-\lambda I_{3}\right) & =\left|\begin{array}{ccc}
\frac{1}{2}|\xi|^{2 \sigma}-\lambda & \frac{1}{2}|\xi|^{2 \sigma}-|\xi|^{2} & -|\xi|^{2} \\
\frac{1}{2}|\xi|^{2 \sigma}+|\xi|^{2} & \frac{1}{2}|\xi|^{2 \sigma}-\lambda & -|\xi|^{2} \\
\frac{1}{2}|\xi|^{2} & \frac{1}{2}|\xi|^{2} & |\xi|^{2}-\lambda
\end{array}\right| \\
& =-\lambda^{3}+\left(|\xi|^{2 \sigma}+|\xi|^{2}\right) \lambda^{2}-\left(2|\xi|^{4}+|\xi|^{2+2 \sigma}\right) \lambda+|\xi|^{6}
\end{aligned}
$$

Plugging $\lambda=i a$ in (2.12) and considering the real and imaginary parts of the coefficient of $a$, we conclude the following two equations, respectively:

$$
\left\{\begin{array} { r l } 
{ - a ^ { 2 } ( | \xi | ^ { 2 \sigma } + | \xi | ^ { 2 } ) + | \xi | ^ { 6 } } & { = 0 , } \\
{ a ( a ^ { 2 } - 2 | \xi | ^ { 4 } - | \xi | ^ { 2 + 2 \sigma } ) } & { = 0 , }
\end{array} \Rightarrow \left\{\begin{array}{l}
a^{2}=\frac{|\xi|^{6}}{|\xi|^{2 \sigma}+|\xi|^{2}} \\
a^{2}=2|\xi|^{4}+|\xi|^{2+2 \sigma}
\end{array}\right.\right.
$$

where we use $a \neq 0$. They lead to a contradiction immediately because $\xi \in Z_{\text {mid }}(\varepsilon, N)$. Then, no pure imaginary characteristic roots of $A(|\xi| ; \sigma)$ for all $\sigma \in[0,1) \cup(1,2]$ can exist for frequencies in the bounded zone. Consequently, due to the compactness of the bounded zone $Z_{\text {mid }}(\varepsilon, N)$ and the continuity of $\operatorname{Re} \lambda_{j}(|\xi|)$ together with $\operatorname{Re} \lambda_{j}(|\xi|)>0, j=1,2,3$, for $|\xi|=\varepsilon$ and $|\xi|=N$, we complete the proof immediately.

\subsection{Representations of solutions}

From Lemmas 2.1 and 2.2, we know that when $\sigma \in[0,1) \cup(1,2]$ with small frequencies or large frequencies the uniform invertibility of $T_{\sigma \text {,int }}$ and $T_{\sigma \text {,ext }}$ hold. Thus, we have the next theorems for the representations of solutions. The proofs of them are based on Lemmas 2.1 and 2.2.

Theorem 2.1. There exists a matrix $T_{\sigma, \text { int }}$ for $\sigma \in[0,1) \cup(1,2]$, which is uniformly invertible for small frequencies such that the following representation formula for the Cauchy problem (2.4) with $\sigma \in[0,1) \cup(1,2]$ holds:

$$
\chi_{\mathrm{int}}(\xi) w^{(0)}(t, \xi)=\chi_{\mathrm{int}}(\xi) T_{\sigma, \text { int }} \operatorname{diag}\left(e^{-\lambda_{1}(|\xi|) t}, e^{-\lambda_{2}(|\xi|) t}, e^{-\lambda_{3}(|\xi|) t}\right) T_{\sigma, \text { int }}^{-1} w_{0}^{(0)}(\xi),
$$

where the characteristic roots $\lambda_{j}(|\xi|)$ for $j=1,2,3$, have the following asymptotic behavior: 
- if $\sigma \in[0,1)$, then we have

$$
\lambda_{1}(|\xi|)=|\xi|^{4-2 \sigma}, \quad \lambda_{2}(|\xi|)=|\xi|^{2}+|\xi|^{4-2 \sigma}, \quad \lambda_{3}(|\xi|)=|\xi|^{2 \sigma}-2|\xi|^{4-2 \sigma},
$$

modulo $\mathrm{O}\left(|\xi|^{6-4 \sigma}\right)$;

- if $\sigma \in(1,2]$, then we have

$$
\lambda_{1}(|\xi|)=y_{1}|\xi|^{2}, \quad \lambda_{2}(|\xi|)=y_{2}|\xi|^{2}, \quad \lambda_{3}(|\xi|)=y_{3}|\xi|^{2},
$$

modulo $\mathcal{O}\left(|\xi|^{2 \sigma}\right)$, where $y_{1}, y_{2}, y_{3}$ are determined in (2.9).

Theorem 2.2. There exists a matrix $T_{\sigma, \operatorname{ext}}$ for $\sigma \in[0,1) \cup(1,2]$, which is uniformly invertible for large frequencies such that the following representation formula for the Cauchy problem (2.4) with $\sigma \in[0,1) \cup(1,2]$ holds:

$$
\chi_{\operatorname{ext}}(\xi) w^{(0)}(t, \xi)=\chi_{\operatorname{ext}}(\xi) T_{\sigma, \operatorname{ext}} \operatorname{diag}\left(e^{-\lambda_{1}(|\xi|) t}, e^{-\lambda_{2}(|\xi|) t}, e^{-\lambda_{3}(|\xi|) t}\right) T_{\sigma, \text { ext }}^{-1} w_{0}^{(0)}(\xi),
$$

where the characteristic roots $\lambda_{j}(|\xi|)$ for $j=1,2,3$, have the following asymptotic behavior:

- if $\sigma \in[0,1)$, then we have

$$
\lambda_{1}(|\xi|)=y_{1}|\xi|^{2}, \quad \lambda_{2}(|\xi|)=y_{2}|\xi|^{2}, \quad \lambda_{3}(|\xi|)=y_{3}|\xi|^{2},
$$

modulo $\mathcal{O}\left(|\xi|^{2 \sigma}\right)$, where $y_{1}, y_{2}, y_{3}$ are determined in (2.9);

- if $\sigma \in(1,2]$, then we have

$$
\lambda_{1}(|\xi|)=|\xi|^{4-2 \sigma}, \quad \lambda_{2}(|\xi|)=|\xi|^{2}+|\xi|^{4-2 \sigma}, \quad \lambda_{3}(|\xi|)=|\xi|^{2 \sigma}-2|\xi|^{4-2 \sigma},
$$

modulo $\Theta\left(|\xi|^{6-4 \sigma}\right)$.

Lastly, considering (2.4) with $\sigma=1$, from Lemma 2.3 we can derive the explicit representation of solutions in the following statement.

Theorem 2.3. There exists a matrix $T_{1,0}$, which is uniformly invertible for all frequencies such that the following representation formula for the Cauchy problem (2.4) with $\sigma=1$ holds:

$$
w^{(0)}(t, \xi)=T_{1,0} \operatorname{diag}\left(e^{-\lambda_{1}(|\xi|) t}, e^{-\lambda_{2}(|\xi|) t}, e^{-\lambda_{3}(|\xi|) t}\right) T_{1,0}^{-1} w_{0}^{(0)}(\xi),
$$

where the characteristic roots $\lambda_{j}(|\xi|)$ have the following explicit expressions:

$$
\lambda_{1}(|\xi|)=y_{4}|\xi|^{2}, \quad \lambda_{2}(|\xi|)=y_{5}|\xi|^{2}, \quad \lambda_{3}(|\xi|)=y_{6}|\xi|^{2},
$$

where $y_{4}, y_{5}, y_{6}$ are determined in (2.11).

\section{Some qualitative properties of solutions}

In this section we derive smoothing effect of solutions and $L^{2}$ well-posedness for linear thermoelastic plate equations with friction or structural damping.

Let us study smoothing effect of solutions initially.

Theorem 3.1. Let us assume $\left(|D|^{2} u_{0}, u_{1}, \theta_{0}\right) \in L^{2}\left(\mathbb{R}^{n}\right) \times L^{2}\left(\mathbb{R}^{n}\right) \times L^{2}\left(\mathbb{R}^{n}\right)$. Then, the solution to the Cauchy problem (1.2) with $\sigma \in[0,2)$ belongs to Gevrey spaces such that

$$
\left(|D|^{s+2} u,|D|^{s} u_{t},|D|^{s} \theta\right)(t, \cdot) \in \Gamma^{\kappa}\left(\mathbb{R}^{n}\right) \times \Gamma^{\kappa}\left(\mathbb{R}^{n}\right) \times \Gamma^{\kappa}\left(\mathbb{R}^{n}\right) \text { for any } t>0,
$$

with $s \geq 0$, where the parameter $\kappa=1$ when $\sigma \in\left[0, \frac{3}{2}\right]$, and $\kappa=\frac{1}{4-2 \sigma}$ when $\sigma \in\left(\frac{3}{2}, 2\right)$. 
Proof. To understand Gevrey smoothing of the solution, we only need to study the regularity properties of the solution for frequencies in the large zone $Z_{\text {ext }}(N)$. From Theorem 2.2 we may estimate

$$
\chi_{\operatorname{ext}}(\xi)|\xi|^{s}\left|w^{(0)}(t, \xi)\right| \lesssim \begin{cases}\chi_{\operatorname{ext}}(\xi)|\xi|^{s} e^{-|\xi|^{2} t}\left|w_{0}^{(0)}(\xi)\right| & \text { if } \sigma \in[0,1] \\ \chi_{\operatorname{ext}}(\xi)|\xi|^{s} e^{-|\xi|^{4-2 \sigma} t}\left|w_{0}^{(0)}(\xi)\right| & \text { if } \sigma \in(1,2)\end{cases}
$$

When we take the parameter $\kappa$ in Gevrey spaces $\Gamma^{\kappa}\left(\mathbb{R}^{n}\right)$ such that $\kappa=1$ if $\sigma \in\left[0, \frac{3}{2}\right]$, and $\kappa=\frac{1}{4-2 \sigma}$ if $\sigma \in\left(\frac{3}{2}, 2\right)$, they lead to

$$
\mathscr{F}_{\xi \rightarrow x}^{-1}\left(|\xi|^{s} w^{(0)}(t, \xi)\right)(t, \cdot) \in \Gamma^{\kappa}\left(\mathbb{R}^{n}\right) \text { for any } t>0 .
$$

One can complete the proof strictly following the paper [29].

Remark 3.1. We notice that for the Cauchy problem (1.2) with $\sigma \in\left[0, \frac{3}{2}\right]$, the solution belongs to the Gevrey space $\Gamma^{1}\left(\mathbb{R}^{n}\right)$, which means analytic smoothing of the solution.

Remark 3.2. Let us consider the Cauchy problem (1.2) with $\sigma=2$. The representation of solutions for large frequencies from Theorem 2.2 implies for $s \geq 0$ that

$$
\chi_{\operatorname{ext}}(\xi)|\xi|^{s}\left|w^{(0)}(t, \xi)\right| \lesssim \chi_{\operatorname{ext}}(\xi) e^{-t}|\xi|^{s}\left|w_{0}^{(0)}(\xi)\right| .
$$

Thus, the solution does not belong to Gevrey spaces $\Gamma^{\kappa}\left(\mathbb{R}^{n}\right)$ with $\kappa \in[1, \infty)$.

Remark 3.3. The statement of Theorem 3.1 tells us that the thermal dissipation generated by Fourier's law has a dominant influence in comparison with friction and structural damping only if $\sigma \in\left[0, \frac{3}{2}\right]$ on Gevrey smoothing. We remark that the threshold of Gevrey smoothing of solutions is $\sigma=\frac{3}{2}$.

After applying the representations of solutions from Theorems 2.1, 2.2 and 2.3, we immediately prove the following $L^{2}$ well-posedness for the Cauchy problem (1.2).

Theorem 3.2. Let us assume $\left(|D|^{2} u_{0}, u_{1}, \theta_{0}\right) \in L^{2}\left(\mathbb{R}^{n}\right) \times L^{2}\left(\mathbb{R}^{n}\right) \times L^{2}\left(\mathbb{R}^{n}\right)$. Then, there exists a uniquely determined solution to the Cauchy problem (1.2) with $\sigma \in[0,2]$, which satisfies

$$
u \in \mathscr{C}\left([0, \infty), \dot{H}^{2}\left(\mathbb{R}^{n}\right)\right), \quad u_{t} \in \mathscr{C}\left([0, \infty), L^{2}\left(\mathbb{R}^{n}\right)\right), \quad \theta \in \mathscr{C}\left([0, \infty), L^{2}\left(\mathbb{R}^{n}\right)\right) .
$$

Remark 3.4. One also can derive $H^{s}$ well-posedness for the Cauchy problem (1.2) for all $s \in \mathbb{R}$.

\section{Estimates for solutions}

This section mainly develops some estimates for solutions to linear thermoelastic plate equations with different damping mechanisms in $\mathbb{R}^{n}, n \geq 1$. The section is organized as follows. First of all, by using phase space analysis and the representations of solutions stated in Theorems 2.1, 2.2, 2.3, we derive estimates of solutions to (1.2) with initial data belonging to $H^{s}\left(\mathbb{R}^{n}\right) \cap L^{m}\left(\mathbb{R}^{n}\right)$ for $s \geq 0$ and $m \in[1,2]$. Moreover, in spired of $[12,14,16]$, we investigate estimates of solutions to (1.2) with initial data taking from wighted $L^{1}$ spaces, i.e., $H^{s}\left(\mathbb{R}^{n}\right) \cap L^{1, \delta}\left(\mathbb{R}^{n}\right)$ for $s \geq 0$ and $\delta \in(0,1]$. Eventually, we study $L^{p}-L^{q}$ estimates not necessary on the conjugate line with the aid of some applications of $L^{r}$ estimates for oscillating integrals. 


\subsection{Energy estimates}

Before stating our main results, let us denote the parameters for $n \geq 1, s \geq 0$ and $m \in[1,2]$ by the following way:

$$
\gamma(\sigma, n, m, s):= \begin{cases}\frac{(2-m) n+2 m s}{4 m(2-\sigma)} & \text { if } \sigma \in[0,1), \\ \frac{(2-m) n+2 m s}{4 m} & \text { if } \sigma \in[1,2] .\end{cases}
$$

It will be used to described the decay rate of the energy estimates later.

Additionally, we define the function spaces $\mathscr{A}_{m, s}\left(\mathbb{R}^{n}\right)$ for $s \geq 0$ and $m \in[1,2]$

$$
\mathcal{A}_{m, s}\left(\mathbb{R}^{n}\right):=\left(H^{s}\left(\mathbb{R}^{n}\right) \cap L^{m}\left(\mathbb{R}^{n}\right)\right) \times\left(H^{s}\left(\mathbb{R}^{n}\right) \cap L^{m}\left(\mathbb{R}^{n}\right)\right) \times\left(H^{s}\left(\mathbb{R}^{n}\right) \cap L^{m}\left(\mathbb{R}^{n}\right)\right),
$$

and the function spaces $\mathscr{B}_{\delta, s}\left(\mathbb{R}^{n}\right)$ for $s \geq 0$ and $\delta \in[0,1]$

$$
\mathscr{B}_{\delta, s}\left(\mathbb{R}^{n}\right):=\left(H^{s}\left(\mathbb{R}^{n}\right) \cap L^{1, \delta}\left(\mathbb{R}^{n}\right)\right) \times\left(H^{s}\left(\mathbb{R}^{n}\right) \cap L^{1, \delta}\left(\mathbb{R}^{n}\right)\right) \times\left(H^{s}\left(\mathbb{R}^{n}\right) \cap L^{1, \delta}\left(\mathbb{R}^{n}\right)\right),
$$

carrying their corresponding norms.

Theorem 4.1. Let us assume $\left(|D|^{2} u_{0}, u_{1}, \theta_{0}\right) \in \mathscr{A}_{2, s}\left(\mathbb{R}^{n}\right)$. Then, the solution to the Cauchy problem (1.2) with $\sigma \in[0,2]$ satisfies the following estimates:

$$
\begin{aligned}
\left\||D|^{2} u(t, \cdot)\right\|_{\dot{H}^{s}\left(\mathbb{R}^{n}\right)} & +\left\|u_{t}(t, \cdot)\right\|_{\dot{H}^{s}\left(\mathbb{R}^{n}\right)}+\|\theta(t, \cdot)\|_{\dot{H}^{s}\left(\mathbb{R}^{n}\right)} \\
& \lesssim(1+t)^{-\gamma(\sigma, n, 2, s)}\left\|\left(|D|^{2} u_{0}, u_{1}, \theta_{0}\right)\right\|_{\mathcal{A}_{2, s}\left(\mathbb{R}^{n}\right)} .
\end{aligned}
$$

Proof. We can complete the proof of Theorem 4.1 by applying the Parseval-Plancherel theorem.

Remark 4.1. Let us assume $\left(|D|^{2} u_{0}, u_{1}, \theta_{0}\right) \in \dot{H}^{s}\left(\mathbb{R}^{n}\right) \times \dot{H}^{s}\left(\mathbb{R}^{n}\right) \times \dot{H}^{s}\left(\mathbb{R}^{n}\right)$ for $s \geq 0$. Then, the solution satisfies the following bounded estimates:

$$
\begin{aligned}
\left\||D|^{2} u(t, \cdot)\right\|_{\dot{H}^{s}\left(\mathbb{R}^{n}\right)} & +\left\|u_{t}(t, \cdot)\right\|_{\dot{H}^{s}\left(\mathbb{R}^{n}\right)}+\|\theta(t, \cdot)\|_{\dot{H}^{s}\left(\mathbb{R}^{n}\right)} \\
& \lesssim\left\|\left(|D|^{2} u_{0}, u_{1}, \theta_{0}\right)\right\|_{\dot{H}^{s}\left(\mathbb{R}^{n}\right) \times \dot{H}^{s}\left(\mathbb{R}^{n}\right) \times \dot{H}^{s}\left(\mathbb{R}^{n}\right)} .
\end{aligned}
$$

Next, we consider initial data taking from $H^{s}$ with additional regularity $L^{m}, m \in[1,2)$, which implies an additional decay in the corresponding estimates.

Theorem 4.2. Let us assume $\left(|D|^{2} u_{0}, u_{1}, \theta_{0}\right) \in \mathscr{A}_{m, s}\left(\mathbb{R}^{n}\right)$, where $s \geq 0$ and $m \in[1,2)$. Then, the solution to the Cauchy problem (1.2) with $\sigma \in[0,2]$ satisfies the next estimates:

$$
\begin{aligned}
\left\||D|^{2} u(t, \cdot)\right\|_{\dot{H}^{s}\left(\mathbb{R}^{n}\right)} & +\left\|u_{t}(t, \cdot)\right\|_{\dot{H}^{s}\left(\mathbb{R}^{n}\right)}+\|\theta(t, \cdot)\|_{\dot{H}^{s}\left(\mathbb{R}^{n}\right)} \\
& \lesssim(1+t)^{-\gamma(\sigma, n, m, s)}\left\|\left(|D|^{2} u_{0}, u_{1}, \theta_{0}\right)\right\|_{\mathcal{A}_{m, s}\left(\mathbb{R}^{n}\right)} .
\end{aligned}
$$

Proof. For frequencies in the small zone, we apply Hölder's inequality and the Hausdorff-Young inequality to get the following estimates:

$$
\begin{aligned}
\left\|\chi_{\mathrm{int}}(\xi)|\xi|^{s} w^{(0)}(t, \xi)\right\|_{L^{2}\left(\mathbb{R}^{n}\right)} \\
\quad \lesssim \begin{cases}\left\|\chi_{\mathrm{int}}(\xi)|\xi|^{s} e^{-c|\xi|^{4-2 \sigma} t}\right\|_{L^{\frac{2 m}{2-m}}\left(\mathbb{R}^{n}\right)}\left\|\mathscr{F}^{-1}\left(w_{0}^{(0)}\right)\right\|_{L^{m}\left(\mathbb{R}^{n}\right)} & \text { if } \sigma \in[0,1), \\
\left\|\chi_{\mathrm{int}}(\xi)|\xi|^{s} e^{-c|\xi|^{2} t}\right\|_{L^{\frac{2 m}{2-m}}\left(\mathbb{R}^{n}\right)}\left\|\mathscr{F}^{-1}\left(w_{0}^{(0)}\right)\right\|_{L^{m}\left(\mathbb{R}^{n}\right)} & \text { if } \sigma \in[1,2],\end{cases} \\
\lesssim \begin{cases}(1+t)^{-\frac{(2-m) n+2 m s}{4 m(2-\sigma)}}\left\|\mathscr{F}^{-1}\left(w_{0}^{(0)}\right)\right\|_{L^{m}\left(\mathbb{R}^{n}\right)} & \text { if } \sigma \in[0,1), \\
(1+t)^{-\frac{(2-m) n+2 m s}{4 m}}\left\|\mathscr{F}^{-1}\left(w_{0}^{(0)}\right)\right\|_{L^{m}\left(\mathbb{R}^{n}\right)} & \text { if } \sigma \in[1,2],\end{cases}
\end{aligned}
$$


where we use the following facts for $\bar{m} \in[1, \infty), \alpha_{0}>0$ and $s \geq 0$ :

$$
\begin{aligned}
&\left\|\chi_{\mathrm{int}}(\xi)|\xi|^{s} e^{-c|\xi|^{\alpha_{0}} t}\right\|_{L^{\bar{m}}\left(\mathbb{R}^{n}\right)}^{\bar{m}} \lesssim 1 \quad \text { if } 0 \leq t \leq 1, \\
&\left\|\chi_{\mathrm{int}}(\xi)|\xi|^{s} e^{-c|\xi|^{\alpha_{0}} t}\right\|_{L^{\bar{m}}\left(\mathbb{R}^{n}\right)}^{\bar{m}}=\int_{0}^{\varepsilon} r^{s \bar{m}+n-1} e^{-c \bar{m} r^{\alpha_{0}} t} d r \\
&=\frac{1}{\alpha_{0}} t^{-\frac{1}{\alpha_{0}}(s \bar{m}+n)} \int_{0}^{\varepsilon^{\alpha_{0}} t} \tau^{\frac{1}{\alpha_{0}}(s \bar{m}+n)-1} e^{-c \bar{m} \tau^{\alpha_{0}}} d \tau \\
& \lesssim t^{-\frac{\bar{m}}{\alpha_{0}}\left(s+\frac{n}{\bar{m}}\right)} \quad \text { if } 1 \leq t .
\end{aligned}
$$

For frequencies in the bounded zone and the large zone, we obtain an exponential decay estimate

$$
\left\|\left(\chi_{\operatorname{mid}}(\xi)+\chi_{\operatorname{ext}}(\xi)\right)|\xi|^{s} w^{(0)}(t, \xi)\right\|_{L^{2}\left(\mathbb{R}^{n}\right)} \lesssim e^{-c t}\left\|\mathscr{F}^{-1}\left(w_{0}^{(0)}\right)\right\|_{H^{s}\left(\mathbb{R}^{n}\right)},
$$

where the constant $c>0$. Finally, combining with the Parseval-Plancherel theorem, the proof of Theorem 4.2 is complete.

Remark 4.2. Concerning the sharpness of the derived energy estimates in Theorem 4.2, we point out that the estimates for $\left\||\xi|^{s} w^{(0)}(t, \xi)\right\|_{L^{2}\left(\mathbb{R}^{n}\right)}$ seen to be sharp because diagonalization procedure is used in deriving representations of solutions.

Next, we discuss energy estimates with initial data taking from the weighted spaces $L^{1, \delta}$ for $\delta \in(0,1]$ (see Notation in Section 1). Before stating our result, we recall the following useful lemma, which was introduced in Lemma 2.1 in the paper [14].

Lemma 4.1. Let $\delta \in(0,1]$ and $f \in L^{1, \delta}\left(\mathbb{R}^{n}\right)$. Then, the following estimate holds:

$$
|\hat{f}(\xi)| \leq C_{\delta}|\xi|^{\delta}\|f\|_{L^{1, \delta}\left(\mathbb{R}^{n}\right)}+\left|\int_{\mathbb{R}^{n}} f(x) d x\right|
$$

with some constant $C_{\delta}>0$.

Theorem 4.3. Let us assume $\left(|D|^{2} u_{0}, u_{1}, \theta_{0}\right) \in \mathscr{B}_{\delta, s}\left(\mathbb{R}^{n}\right)$, where $s \geq 0$ and $\delta \in(0,1]$. Then, the solution the Cauchy problem (1.2) with $\sigma \in[0,2]$ satisfies the following estimates:

$$
\begin{aligned}
\left\||D|^{2} u(t, \cdot)\right\|_{\dot{H}^{s}\left(\mathbb{R}^{n}\right)}+\left\|u_{t}(t, \cdot)\right\|_{\dot{H}^{s}\left(\mathbb{R}^{n}\right)}+\|\theta(t, \cdot)\|_{\dot{H}^{s}\left(\mathbb{R}^{n}\right)} \\
\quad \lesssim(1+t)^{-\gamma(\sigma, n, 1, s+\delta)}\left\|\left(|D|^{2} u_{0}, u_{1}, \theta_{0}\right)\right\|_{\mathscr{B}_{\delta, s}\left(\mathbb{R}^{n}\right)}+(1+t)^{-\gamma(\sigma, n, 1, s)}\left|\int_{\mathbb{R}^{n}} U_{0}(x) d x\right|
\end{aligned}
$$

where initial data $U_{0}(x)$ is defined in (2.6).

Proof. One can prove Theorem 4.3 strictly following Theorem 4.3 in the recent paper [2].

Remark 4.3. By restricting

$$
\left|\int_{\mathbb{R}^{n}} U_{0}(x) d x\right|=0
$$

we find that the decay rate given in Theorem 4.2 when $m=1$ can be improved by $(1+t)^{-\frac{\delta}{2}}$ for $\delta \in(0,1]$. We need to point out that the additional condition (4.13) holds when $U_{0}(x)$ is odd function with respect to $x_{n}$, in other words,

$$
U_{0}\left(x_{1}, \ldots, x_{n-1},-x_{n}\right)=-U_{0}\left(x_{1}, \ldots, x_{n-1}, x_{n}\right) .
$$

Remark 4.4. The statements of Theorems 4.1, 4.2 and 4.3 tell us that the thermal dissipation generated by Fourier's law has a dominant influence in comparison with friction and structural damping only if $\sigma \in[1,2]$ on energy estimates. 


\section{2. $L^{p}-L^{q}$ estimates not necessary on the conjugate line}

In the beginning, let us introduce the parameters to depict the decay rate

$$
\mu(\sigma, n, p, q, s):= \begin{cases}\frac{s}{4-2 \sigma}+\frac{n}{4-2 \sigma}\left(\frac{1}{p}-\frac{1}{q}\right) & \text { if } \sigma \in[0,1), \\ \frac{s}{2}+\frac{n}{2}\left(\frac{1}{p}-\frac{1}{q}\right) & \text { if } \sigma \in[1,2],\end{cases}
$$

where $s \geq 0$ and $1 \leq p \leq q \leq \infty$.

Moreover, we define the parameter to depict the regularity for initial data

$$
M_{n, s, p, q}>s+n\left(\frac{1}{p}-\frac{1}{q}\right),
$$

where $s \geq 0$ and $1 \leq p \leq 2 \leq q \leq \infty$.

4.2.1. $L^{p}-L^{q}$ estimates for the model with $\sigma \in[0,1) \cup(1,2]$. Before starting our main theorem, we prove the following useful lemma first.

Lemma 4.2. Let us $f \in \mathcal{S}\left(\mathbb{R}^{n}\right)$ and $\kappa_{1}>0, \kappa_{2} \geq 0, s \geq 0$. Then, the next estimates hold:

$$
\begin{aligned}
& \left\|\mathscr{F}_{\xi \rightarrow x}^{-1}\left(\chi_{\operatorname{int}}(\xi)|\xi|^{s} e^{-c|\xi|^{\kappa_{1}} t} \hat{f}(\xi)\right)\right\|_{L^{q}\left(\mathbb{R}^{n}\right)} \lesssim(1+t)^{-\frac{s}{\kappa_{1}}-\frac{n}{\kappa_{1}}\left(\frac{1}{p}-\frac{1}{q}\right)}\|f\|_{L^{p}\left(\mathbb{R}^{n}\right)}, \\
& \left\|\mathscr{F}_{\xi \rightarrow x}^{-1}\left(\chi_{\operatorname{ext}}(\xi)|\xi|^{s} e^{-c|\xi|^{\kappa_{2}} t} \hat{f}(\xi)\right)\right\|_{L^{q}\left(\mathbb{R}^{n}\right)} \lesssim e^{-c t}\left\|\langle D\rangle^{M_{n, s, p, q}} f\right\|_{L^{p}\left(\mathbb{R}^{n}\right)},
\end{aligned}
$$

where $c>0,1 \leq p \leq 2 \leq q \leq \infty$ and $M_{n, s, p, q}$ is chosen in (4.15).

Proof. Let us prove (4.16) first. Applying the Hausdorff-Young inequality yields

$$
\left\|\mathscr{F}_{\xi \rightarrow x}^{-1}\left(\chi_{\mathrm{int}}(\xi)|\xi|^{s} e^{-c|\xi|^{\kappa_{1}} t} \hat{f}(\xi)\right)\right\|_{L^{q}\left(\mathbb{R}^{n}\right)} \lesssim\left\|\chi_{\mathrm{int}}(\xi)|\xi|^{s} e^{-c|\xi|^{\kappa_{1}} t} \hat{f}(\xi)\right\|_{L^{q^{\prime}\left(\mathbb{R}^{n}\right)}} .
$$

Here $\frac{1}{q}+\frac{1}{q^{\prime}}=1$ with $2 \leq q \leq \infty$. By Hölder's inequality, the estimate holds

$$
\left\|\chi_{\mathrm{int}}(\xi)|\xi|^{s} e^{-c|\xi|^{\kappa_{1}} t} \hat{f}(\xi)\right\|_{L^{q^{\prime}\left(\mathbb{R}^{n}\right)}} \lesssim\left\|\chi_{\mathrm{int}}(\xi)|\xi|^{s} e^{-c|\xi|^{\kappa_{1}} t}\right\|_{L^{\tilde{p}}\left(\mathbb{R}^{n}\right)}\|\hat{f}\|_{L^{p^{\prime}\left(\mathbb{R}^{n}\right)}},
$$

where $\frac{1}{q^{\prime}}=\frac{1}{\tilde{p}}+\frac{1}{p^{\prime}}$ with $2 \leq p^{\prime} \leq \infty$. Finally, combining with (4.18), (4.19) and the Hausdorff-Young inequality leads to

$$
\left\|\mathscr{F}_{\xi \rightarrow x}^{-1}\left(\chi_{\operatorname{int}}(\xi)|\xi|^{s} e^{-c|\xi|^{\kappa_{1}} t} \hat{f}(\xi)\right)\right\|_{L^{q}\left(\mathbb{R}^{n}\right)} \lesssim(1+t)^{-\frac{s}{\kappa_{1}}-\frac{n}{\kappa_{1}}\left(\frac{1}{p}-\frac{1}{q}\right)}\|f\|_{L^{p}\left(\mathbb{R}^{n}\right)} .
$$

Next, we begin with proving (4.17). For $0 \leq t \leq 1$, by the similar approach we have

$$
\begin{aligned}
& \left\|\mathscr{F}_{\xi \rightarrow x}^{-1}\left(\chi_{\operatorname{ext}}(\xi)|\xi|^{s} e^{-c|\xi|^{\kappa_{2}} t} \hat{f}(\xi)\right)\right\|_{L^{q}\left(\mathbb{R}^{n}\right)} \lesssim\left\|\chi_{\mathrm{ext}}(\xi)\langle\xi\rangle^{s} \hat{f}(\xi)\right\|_{L^{q^{\prime}\left(\mathbb{R}^{n}\right)}} \\
& \quad \lesssim\left\|\chi_{\operatorname{ext}}(\xi)\langle\xi\rangle^{-n\left(\frac{1}{p}-\frac{1}{q}\right)-\epsilon}\right\|_{L^{\tilde{p}\left(\mathbb{R}^{n}\right)}}\left\|\chi_{\mathrm{ext}}(\xi)\langle\xi\rangle^{s+n\left(\frac{1}{p}-\frac{1}{q}\right)+\epsilon} \hat{f}(\xi)\right\|_{L^{p^{\prime}\left(\mathbb{R}^{n}\right)}},
\end{aligned}
$$

where $\frac{1}{q}+\frac{1}{q^{\prime}}=1, \frac{1}{q^{\prime}}=\frac{1}{\tilde{p}}+\frac{1}{p^{\prime}}$ with $2 \leq q \leq \infty, 2 \leq p^{\prime} \leq \infty$ and $\epsilon>0$.

The following fact holds:

$$
\left\|\chi_{\operatorname{ext}}(\xi)\langle\xi\rangle^{-n\left(\frac{1}{p}-\frac{1}{q}\right)-\epsilon}\right\|_{L^{\tilde{p}}\left(\mathbb{R}^{n}\right)}^{\tilde{p}}=\int_{N}^{\infty}\langle r\rangle^{-n\left(\frac{1}{p}-\frac{1}{q}\right) \tilde{p}-\epsilon \tilde{p}+n-1} d r=\int_{N}^{\infty}\langle r\rangle^{-\epsilon \tilde{p}-1} d r<\infty .
$$

Summarizing (4.20), (4.21) and using the Hausdorff-Young inequality we derive

$$
\left\|\mathscr{F}_{\xi \rightarrow x}^{-1}\left(\chi_{\operatorname{ext}}(\xi)|\xi|^{s} e^{-c|\xi|^{\kappa_{2}} t} \hat{f}(\xi)\right)\right\|_{L^{q}\left(\mathbb{R}^{n}\right)} \lesssim\left\|\langle D\rangle^{s+n\left(\frac{1}{p}-\frac{1}{q}\right)+\epsilon} f\right\|_{L^{p}\left(\mathbb{R}^{n}\right)}
$$

for $1 \leq p \leq 2 \leq q \leq \infty$ and $0 \leq t \leq 1$.

For the case $t \geq 1$, according to $|\bar{\xi}| \geq N$ we may obtain

$$
\left\|\mathscr{F}_{\xi \rightarrow x}^{-1}\left(\chi_{\operatorname{ext}}(\xi)|\xi|^{s} e^{-c|\xi|^{\kappa_{2}} t} \hat{f}(\xi)\right)\right\|_{L^{q}\left(\mathbb{R}^{n}\right)} \lesssim e^{-c t}\left\|\langle D\rangle^{s+n\left(\frac{1}{p}-\frac{1}{q}\right)+\epsilon} f\right\|_{L^{p}\left(\mathbb{R}^{n}\right)} .
$$


Hence, the proof of Lemma 4.2 is completed.

Now, let us derive $L^{p}-L^{q}$ estimates of solutions to the Cauchy problem (1.2) with $\sigma \in[0,1) \cup(1,2]$, where $1 \leq p \leq 2 \leq q \leq \infty$.

Theorem 4.4. Let us assume $\left(|D|^{2} u_{0}, u_{1}, \theta_{0}\right) \in \mathcal{S}\left(\mathbb{R}^{n}\right) \times \mathcal{S}\left(\mathbb{R}^{n}\right) \times \mathcal{S}\left(\mathbb{R}^{n}\right)$. Then, the solution to the Cauchy problem (1.2) with $\sigma \in[0,1) \cup(1,2]$ satisfies the following estimates:

$$
\begin{aligned}
& \left\||D|^{2} u(t, \cdot)\right\|_{\dot{H}_{q}^{s}\left(\mathbb{R}^{n}\right)}+\left\|u_{t}(t, \cdot)\right\|_{\dot{H}_{q}^{s}\left(\mathbb{R}^{n}\right)}+\|\theta(t, \cdot)\|_{\dot{H}_{q}^{s}\left(\mathbb{R}^{n}\right)} \\
& \quad \lesssim(1+t)^{-\mu(\sigma, n, p, q, s)}\left\|\left(|D|^{2} u_{0}, u_{1}, \theta_{0}\right)\right\|_{H_{p}^{M_{n, s, p, q}}\left(\mathbb{R}^{n}\right) \times H_{p}^{M_{n, s, p, q}}\left(\mathbb{R}^{n}\right) \times H_{p}^{M_{n, s, p, q}}\left(\mathbb{R}^{n}\right)},
\end{aligned}
$$

with $s \geq 0,1 \leq p \leq 2 \leq q \leq \infty$ and $M_{n, s, p, q}>s+n\left(\frac{1}{p}-\frac{1}{q}\right)$.

Remark 4.5. If one is interested in the case $p \in(1,2]$, then we can choose $M_{n, s, p, q}=s+n\left(\frac{1}{p}-\frac{1}{q}\right)$.

Proof. From Theorems 2.1, 2.3, 2.3 and Lemma 2.4 we obtain

$$
\begin{aligned}
& \left\||D|^{s} \mathscr{F}_{\xi \rightarrow x}^{-1}\left(w^{(0)}\right)(t, \cdot)\right\|_{L^{q}\left(\mathbb{R}^{n}\right)} \lesssim\left\||\xi|^{s} w^{(0)}(t, \xi)\right\|_{L^{q^{\prime}\left(\mathbb{R}^{n}\right)}} \\
& \lesssim\left\|\chi_{\operatorname{int}}(\xi)|\xi|^{s} w^{(0)}(t, \xi)\right\|_{L^{q^{\prime}\left(\mathbb{R}^{n}\right)}}+\left\|\chi_{\operatorname{mid}}(\xi)|\xi|^{s} w^{(0)}(t, \xi)\right\|_{L^{q^{\prime}}\left(\mathbb{R}^{n}\right)} \\
& \quad+\left\|\chi_{\operatorname{ext}}(\xi)|\xi|^{s} w^{(0)}(t, \xi)\right\|_{L^{q^{\prime}}\left(\mathbb{R}^{n}\right)}
\end{aligned}
$$

where $\frac{1}{q}+\frac{1}{q^{\prime}}=1$ with $2 \leq q \leq \infty$.

Following all steps from Lemma 4.2 we immediately complete the proof.

4.2.2. $L^{p}-L^{q}$ estimates for the model with $\sigma=1$. Due to the treatment in Lemma 2.3, it allows us to obtain explicit representation of solutions. Therefore, it is helpful for us to derive $L^{p}-L^{q}$ estimates of solutions to the Cauchy problem (1.2), where $1 \leq p \leq q \leq \infty$. To do this, let us introduce some results in $L^{p}$ estimates for some oscillating integral by using modified Bessel functions (c.f. [8, 28]).

Lemma 4.3. Let $p \in[1, \infty]$ and $c_{1}>0, c_{2} \neq 0$. Then, the following estimates hold for any $t>0$ :

$$
\begin{aligned}
& \left\|\mathscr{F}_{\xi \rightarrow x}^{-1}\left(|\xi|^{s} e^{-c_{1}|\xi|^{2} t} \sin \left(c_{2}|\xi|^{2} t\right)\right)(t, \cdot)\right\|_{L^{p}\left(\mathbb{R}^{n}\right)} \lesssim t^{-\frac{s}{2}-\frac{n}{2}\left(1-\frac{1}{p}\right)}, \\
& \left\|\mathscr{F}_{\xi \rightarrow x}^{-1}\left(|\xi|^{s} e^{-c_{1}|\xi|^{2} t} \cos \left(c_{2}|\xi|^{2} t\right)\right)(t, \cdot)\right\|_{L^{p}\left(\mathbb{R}^{n}\right)} \lesssim t^{-\frac{s}{2}-\frac{n}{2}\left(1-\frac{1}{p}\right)},
\end{aligned}
$$

where $s \geq 0$ and $n \geq 1$.

Proof. For the proof of (4.23), one can see Proposition 12 in [28]. One can prove (4.22) by some minor modifications of the proof of Proposition 12 in [28].

Theorem 4.5. Let us assume $\left(|D|^{2} u_{0}, u_{1}, \theta_{0}\right) \in L^{p}\left(\mathbb{R}^{n}\right) \times L^{p}\left(\mathbb{R}^{n}\right) \times L^{p}\left(\mathbb{R}^{n}\right)$, where $p \geq 1$. Then, the solution to the Cauchy problem (1.2) with $\sigma=1$ satisfies the next estimates:

$$
\begin{aligned}
& \left\||D|^{2} u(t, \cdot)\right\|_{\dot{H}_{q}^{s}\left(\mathbb{R}^{n}\right)}+\left\|u_{t}(t, \cdot)\right\|_{\dot{H}_{q}^{s}\left(\mathbb{R}^{n}\right)}+\|\theta(t, \cdot)\|_{\dot{H}_{q}^{s}\left(\mathbb{R}^{n}\right)} \\
& \quad \lesssim t^{-\mu(1, n, p, q, s)}\left\|\left(|D|^{2} u_{0}, u_{1}, \theta_{0}\right)\right\|_{L^{p}\left(\mathbb{R}^{n}\right) \times L^{p}\left(\mathbb{R}^{n}\right) \times L^{p}\left(\mathbb{R}^{n}\right)},
\end{aligned}
$$

where $s \geq 0$ and $1 \leq p \leq q \leq \infty$. 
Proof. From Theorem 2.3, the solutions to (1.2) can be explicitly represented by the following way:

$$
\begin{aligned}
\left(u_{t}+|D|^{2}\right. & \left.u, u_{t}-|D|^{2} u, \theta\right)^{\mathrm{T}}(t, x) \\
& =\left(\sum_{j, k=1}^{3} c_{j k l} \mathscr{F}_{\xi \rightarrow x}^{-1}\left(e^{-\operatorname{Re} y_{j+3}|\xi|^{2} t-i \operatorname{Im} y_{j+3}|\xi|^{2} t}\right) *{ }_{(x)} U_{0, k}(x)\right)_{l=1}^{3} \\
& =\left(\sum_{j, k=1}^{3} c_{j k l}\left(K_{0}^{(j)}(t, x)+K_{1}^{(j)}(t, x)\right) *_{(x)} U_{0, k}(x)\right)_{l=1}^{3},
\end{aligned}
$$

where $c_{j k l}$ are constants and the kernels are

$$
\begin{aligned}
K_{0}^{(j)} & :=\mathscr{F}_{\xi \rightarrow x}^{-1}\left(-i \sin \left(\operatorname{Im} y_{j+3}|\xi|^{2} t\right) e^{-\operatorname{Re} y_{j+3}|\xi|^{2} t}\right), \\
K_{1}^{(j)} & :=\mathscr{F}_{\xi \rightarrow x}^{-1}\left(\cos \left(\operatorname{Im} y_{j+3}|\xi|^{2} t\right) e^{-\operatorname{Re} y_{j+3}|\xi|^{2} t}\right) .
\end{aligned}
$$

By applying Lemma 4.3 we get

$$
\sum_{j=1}^{3}\left\||D|^{s} K_{0}^{(j)}(t, \cdot)\right\|_{L^{r}\left(\mathbb{R}^{n}\right)}+\sum_{j=1}^{3}\left\||D|^{s} K_{1}^{(j)}(t, \cdot)\right\|_{L^{r}\left(\mathbb{R}^{n}\right)} \lesssim t^{-\frac{s}{2}-\frac{n}{2}\left(1-\frac{1}{r}\right)}
$$

for all $r \in[1, \infty]$. Then, we directly apply Young's inequality in (4.24) to complete the proof.

We find that if $p=q$ in Theorem 4.5 and we suppose that higher regularity for initial data, the singularity as $t \rightarrow+0$ will disappear. So, we have the next result.

Corollary 4.1. Let us assume $\left(|D|^{2} u_{0}, u_{1}, \theta_{0}\right) \in \dot{H}_{p}^{s}\left(\mathbb{R}^{n}\right) \times \dot{H}_{p}^{s}\left(\mathbb{R}^{n}\right) \times \dot{H}_{p}^{s}\left(\mathbb{R}^{n}\right)$, where $p \geq 1$ and $s \geq 0$. Then, the solution to the Cauchy problem (1.2) with $\sigma=1$ satisfies the following bounded estimates:

$$
\begin{aligned}
\left\||D|^{2} u(t, \cdot)\right\|_{\dot{H}_{p}^{s}\left(\mathbb{R}^{n}\right)} & +\left\|u_{t}(t, \cdot)\right\|_{\dot{H}_{p}^{s}\left(\mathbb{R}^{n}\right)}+\|\theta(t, \cdot)\|_{\dot{H}_{p}^{s}\left(\mathbb{R}^{n}\right)} \\
& \lesssim\left\|\left(|D|^{2} u_{0}, u_{1}, \theta_{0}\right)\right\|_{\dot{H}_{p}^{s}\left(\mathbb{R}^{n}\right) \times \dot{H}_{p}^{s}\left(\mathbb{R}^{n}\right) \times \dot{H}_{p}^{s}\left(\mathbb{R}^{n}\right)} .
\end{aligned}
$$

Remark 4.6. One can apply Theorem 4.5 for $t>t_{0} \gg 1$ and Corollary 4.1 for $0 \leq t \leq t_{0}$ to obtain decay estimates for

$$
\left\||D|^{2} u(t, \cdot)\right\|_{\dot{H}_{q}^{s}\left(\mathbb{R}^{n}\right)}+\left\|u_{t}(t, \cdot)\right\|_{\dot{H}_{q}^{s}\left(\mathbb{R}^{n}\right)}+\|\theta(t, \cdot)\|_{\dot{H}_{q}^{s}\left(\mathbb{R}^{n}\right)}
$$

with decay rate $(1+t)^{-\mu(1, n, p, q, s)}$. At this time, initial data should belong to the function spaces $\dot{H}_{q}^{s}\left(\mathbb{R}^{n}\right) \cap L^{p}\left(\mathbb{R}^{n}\right)$, where $n \geq 1,1 \leq p \leq q \leq \infty$ and $s \geq 0$.

Remark 4.7. The statements of Theorems 4.4 and 4.5 indicate that the thermal dissipation generated by Fourier's law has a dominant influence in comparison with friction and structural damping only if $\sigma \in[1,2]$ on $L^{p}-L^{q}$ estimates away the conjugate line.

\section{Diffusion phenomena}

It is well known that diffusion phenomena allow one to bridge the decay behavior of the solution to (1.2) with the solution for the corresponding evolution. It also provides a tool to tackle the asymptotic profiles of solutions. In this section we study diffusion phenomena of solutions to the Cauchy problem (1.2) for $\sigma \in[0,1) \cup(1,2]$ with initial data carrying different assumptions on the regularity.

In the view of the derived estimates of solutions in Section 4, we find that the decay rate of estimates of solutions are determined by the behavior of the characteristic roots for $\xi \in Z_{\text {int }}(\varepsilon)$ only. For $\xi \in Z_{\text {mid }}(\varepsilon, N) \cup Z_{\text {ext }}(N)$, the solutions satisfies an exponential decay when we assume initial data having suitable regularities. For this reason, we explain diffusion phenomena of solutions for small frequencies in this section. 
Remark 5.1. Considering $\sigma=1$ in the system (2.4), we find that $e^{-y_{j}|\xi|^{2} t}$ with $y_{j} \in \mathbb{C}$ for $j=4,5,6$, plays a determined role in the explicit representation of $w^{(0)}(t, \xi)$ from Theorem 2.3. Then, there is not any improvement in the decay estimates for the difference between the solutions to the system (2.4) with $\sigma=1$ and the solutions to its reference system. Hence, we explain diffusion phenomena for $\sigma \in[0,1) \cup(1,2]$ only.

\subsection{Diffusion phenomena for the model with $\sigma \in[0,1)$}

To describe diffusion phenomena of the solutions to the Cauchy problem (2.4) with $\sigma \in[0,1)$, we consider the following reference system:

$$
\begin{cases}\tilde{u}_{t}+\operatorname{diag}\left((-\Delta)^{2-\sigma},(-\Delta),(-\Delta)^{\sigma}\right) \tilde{u}=0, & t>0, x \in \mathbb{R}^{n}, \\ \tilde{u}(0, x)=\mathscr{F}^{-1}\left(T_{1 \frac{1}{2}}^{-1} T_{1}^{-1} T_{0}^{-1} w_{0}^{(0)}(\xi)\right)(x), & x \in \mathbb{R}^{n},\end{cases}
$$

where $\tilde{u}=\left(\tilde{u}^{(1)}, \tilde{u}^{(2)}, \tilde{u}^{(3)}\right)^{\mathrm{T}}$ and $T_{0}, T_{1}, T_{1 \frac{1}{2}}$ are defined in Lemma 2.1. By applying the partial Fourier transform $\tilde{w}(t, \xi)=\mathscr{F}_{x \rightarrow \xi}(\tilde{u}(t, x)),(5.27)$ can be transformed to

$$
\begin{cases}\tilde{w}_{t}+\operatorname{diag}\left(|\xi|^{4-2 \sigma},|\xi|^{2},|\xi|^{2 \sigma}\right) \tilde{w}=0, & t>0, \xi \in \mathbb{R}^{n}, \\ \tilde{w}(0, \xi)=T_{1 \frac{1}{2}}^{-1} T_{1}^{-1} T_{0}^{-1} w_{0}^{(0)}(\xi), & \xi \in \mathbb{R}^{n}\end{cases}
$$

We know that the solution $\tilde{w}=\tilde{w}(t, \xi)$ to $(5.28)$ can be explicitly represented by

$$
\tilde{w}(t, \xi)=\operatorname{diag}\left(e^{-|\xi|^{4-2 \sigma} t}, e^{-|\xi|^{2} t}, e^{-|\xi|^{2 \sigma} t}\right) T_{1 \frac{1}{2}}^{-1} T_{1}^{-1} T_{0}^{-1} w_{0}^{(0)}(\xi) .
$$

Remark 5.2. According to the evolution system (5.27) with $\sigma=0$, we find that the reference system is consisted of two different evolution equations such that

$$
\begin{array}{ll}
\text { fourth-order parabolic equation equation: } & \tilde{u}_{t}^{(1)}+\Delta^{2} \tilde{u}^{(1)}=0, \\
& \tilde{u}_{t}^{(2)}-\Delta \tilde{u}^{(2)}=0 .
\end{array}
$$

Therefore, we obtain double diffusion phenomena of solution to (1.2) with $\sigma=0$. The effect of double diffusion phenomena was introduced in the recent papers [4, 3].

Remark 5.3. Let us consider (1.2) with $\sigma \in(0,1)$. Inspiring from the dominant asymptotic behavior of eigenvalues such that

$$
\lambda_{1}(|\xi|)=\mathcal{O}\left(|\xi|^{4-2 \sigma}\right), \quad \lambda_{2}(|\xi|)=\mathcal{O}\left(|\xi|^{2}\right), \quad \lambda_{3}(|\xi|)=\mathcal{O}\left(|\xi|^{2 \sigma}\right)
$$

for $\xi \in Z_{\text {int }}(\varepsilon)$, we observe that the evolution system (5.27) is consisted of three different evolution equations, which are

fractional heat equation 1: $\tilde{u}_{t}^{(1)}+(-\Delta)^{2-\sigma} \tilde{u}^{(1)}=0$,

heat equation: $\quad \tilde{u}_{t}^{(2)}-\Delta \tilde{u}^{(2)}=0$,

fractional heat equation 2: $\tilde{u}_{t}^{(3)}+(-\Delta)^{\sigma} \tilde{u}^{(3)}=0$.

We may interpret this effect as triple diffusion phenomena, which is a nature generalization of the effect of double diffusion phenomena.

Theorem 5.1. Let us consider the Cauchy problem (2.4) with $\sigma \in[0,1)$. We assume $\left(|D|^{2} u_{0}, u_{1}, \theta_{0}\right) \in$ $L^{m}\left(\mathbb{R}^{n}\right) \times L^{m}\left(\mathbb{R}^{n}\right) \times L^{m}\left(\mathbb{R}^{n}\right)$ with $m \in[1,2]$. Then, we have the following refinement estimates:

$$
\begin{aligned}
\| \chi_{\text {int }}(D) & \mathscr{F}_{\xi \rightarrow x}^{-1}\left(w^{(0)}-T_{0} T_{1} T_{1 \frac{1}{2}} \tilde{w}\right)(t, \cdot) \|_{\dot{H}^{s}\left(\mathbb{R}^{n}\right)} \\
& \lesssim(1+t)^{-\frac{(2-m) n+2 m s}{4 m(2-\sigma)}-\frac{1-\sigma}{2-\sigma}}\left\|\left(|D|^{2} u_{0}, u_{1}, \theta_{0}\right)\right\|_{L^{m}\left(\mathbb{R}^{n}\right) \times L^{m}\left(\mathbb{R}^{n}\right) \times L^{m}\left(\mathbb{R}^{n}\right)},
\end{aligned}
$$

where $T_{0}, T_{1}, T_{1 \frac{1}{2}}$ are defined in Lemma 2.1. 
Proof. According to the representations of solutions for small frequencies in Theorem 2.1 and the definition of the matrices in (2.8), we may obtain

$$
\chi_{\mathrm{int}}(\xi)|\xi|^{s}\left(w^{(0)}-T_{0} T_{1} T_{1 \frac{1}{2}} \tilde{w}\right)(t, \xi)=\chi_{\mathrm{int}}(\xi)|\xi|^{s}\left(J_{1}(t,|\xi|)+J_{2}(t,|\xi|)+J_{3}(t,|\xi|)\right)
$$

where

$$
\begin{aligned}
& J_{0}(t,|\xi|)=\operatorname{diag}\left(e^{-\lambda_{1}(|\xi|) t}-e^{-|\xi|^{4-2 \sigma} t}, e^{-\lambda_{2}(|\xi|) t}-e^{-|\xi|^{2} t}, e^{-\lambda_{3}(|\xi|) t}-e^{-|\xi|^{2 \sigma} t}\right), \\
& J_{1}(t,|\xi|)=T_{0} T_{1} T_{1 \frac{1}{2}} J_{0}(t,|\xi|) T_{1 \frac{1}{2}}^{-1} T_{1}^{-1} T_{0}^{-1} w_{0}^{(0)}(\xi) \\
& J_{2}(t,|\xi|)=T_{0} T_{1} T_{1 \frac{1}{2}} N_{2}(|\xi|) \operatorname{diag}\left(e^{-\lambda_{1}(|\xi|) t}, e^{-\lambda_{2}(|\xi|) t}, e^{-\lambda_{3}(|\xi|) t}\right) T_{2}^{-1} T_{1}^{-1} T_{0}^{-1} w_{0}^{(0)}(\xi), \\
& J_{3}(t,|\xi|)=-T_{0} T_{1} T_{1 \frac{1}{2}} T_{2} \operatorname{diag}\left(e^{-\lambda_{1}(|\xi|) t}, e^{-\lambda_{2}(|\xi|) t}, e^{-\lambda_{3}(|\xi|) t}\right) T_{2}^{-1} N_{2}(|\xi|) T_{1}^{-1} T_{0}^{-1} w_{0}^{(0)}(\xi),
\end{aligned}
$$

with $N_{2}(|\xi|)=\sigma\left(|\xi|^{2-2 \sigma}\right)$ for $\sigma \in[0,1)$.

Let us define

$$
\begin{aligned}
& h_{1}(|\xi|)=|\xi|^{4-2 \sigma}, \quad h_{2}(|\xi|)=|\xi|^{2}, \quad h_{3}(|\xi|)=|\xi|^{2 \sigma}, \\
& g_{1}(|\xi|)=\lambda_{1}(|\xi|)-h_{1}(|\xi|), \quad g_{2}(|\xi|)=\lambda_{2}(|\xi|)-h_{2}(|\xi|), \quad g_{3}(|\xi|)=\lambda_{3}(|\xi|)-h_{3}(|\xi|) .
\end{aligned}
$$

Applying the following formula for $j=1,2,3$ :

$$
e^{-h_{j}(|\xi|) t+g_{j}(|\xi|) t}-e^{-h_{j}(|\xi|) t}=-g_{j}(|\xi|) t e^{-h_{j}(|\xi|) t} \int_{0}^{1} e^{-g_{j}(|\xi|) t \tau} d \tau
$$

we can get

$$
\begin{aligned}
\| \chi_{\mathrm{int}}(\xi)|\xi|^{s} & \left(w^{(0)}-T_{0} T_{1} T_{1 \frac{1}{2}} \tilde{w}\right)(t, \xi) \|_{L^{2}\left(\mathbb{R}^{n}\right)} \\
= & \left\|\chi_{\mathrm{int}}(\xi)|\xi|^{s}\left(J_{1}(t,|\xi|)+J_{2}(t,|\xi|)+J_{3}(t,|\xi|)\right)\right\|_{L^{2}\left(\mathbb{R}^{n}\right)} \\
& \lesssim\left\|\chi_{\mathrm{int}}(\xi)|\xi|^{s+2-2 \sigma} e^{-|\xi|^{4-2 \sigma} t}\right\|_{L^{\frac{2 m}{2-m}}\left(\mathbb{R}^{n}\right)}\left\|\mathscr{F}^{-1}\left(w_{0}^{(0)}\right)\right\|_{L^{m}\left(\mathbb{R}^{n}\right)} \\
& \lesssim(1+t)^{-\gamma(\sigma, n, m, s)-\frac{1-\sigma}{2-\sigma}}\left\|\mathscr{F}^{-1}\left(w_{0}^{(0)}\right)\right\|_{L^{m}\left(\mathbb{R}^{n}\right)} .
\end{aligned}
$$

Thus, the proof is complete.

Theorem 5.2. Let us consider the Cauchy problem (2.4) with $\sigma \in[0,1)$. We assume $\left(|D|^{2} u_{0}, u_{1}, \theta_{0}\right) \in$ $\delta\left(\mathbb{R}^{n}\right) \times \mathcal{S}\left(\mathbb{R}^{n}\right) \times \mathcal{S}\left(\mathbb{R}^{n}\right)$. Then, we have the following refinement estimates:

$$
\begin{aligned}
\| \chi_{\mathrm{int}}(D) & \mathscr{F}_{\xi \rightarrow x}^{-1}\left(w^{(0)}-T_{0} T_{1} T_{1 \frac{1}{2}} \tilde{w}\right)(t, \cdot) \|_{\dot{H}_{q}^{s}\left(\mathbb{R}^{n}\right)} \\
& \lesssim(1+t)^{-\frac{s}{4-2 \sigma}-\frac{n}{4-2 \sigma}\left(\frac{1}{p}-\frac{1}{q}\right)-\frac{1-\sigma}{2-\sigma}}\left\|\left(|D|^{2} u_{0}, u_{1}, \theta_{0}\right)\right\|_{L^{p}\left(\mathbb{R}^{n}\right) \times L^{p}\left(\mathbb{R}^{n}\right) \times L^{p}\left(\mathbb{R}^{n}\right)},
\end{aligned}
$$

where $T_{0}, T_{1}, T_{1 \frac{1}{2}}$ are defined in Lemma 2.1 with $s \geq 0,1 \leq p \leq 2 \leq q \leq \infty$.

Proof. We may prove this result immediately by using Lemma 4.2.

Remark 5.4. From the statements of Theorems 5.1 and 5.2, we know when $\sigma \in[0,1)$ the thermal dissipation generated by Fourier's law and friction or structural damping have the influence on the reference system at the same time. However, the friction and structural damping have a dominant influence on the decay rate of the estimates. 
5.2. Diffusion phenomena for the model with $\sigma \in(1,2]$

Now, we describe diffusion phenomena of the solutions to the Cauchy problem $(2.4)$ with $\sigma \in(1,2]$ by the reference system as follows:

$$
\begin{cases}\tilde{u}_{t}-\operatorname{diag}\left(y_{1}, y_{2}, y_{3}\right) \Delta \tilde{u}=0, & t>0, x \in \mathbb{R}^{n}, \\ \tilde{u}(0, x)=\mathscr{F}^{-1}\left(T_{0}^{-1} w_{0}^{(0)}(\xi)\right)(x), & x \in \mathbb{R}^{n},\end{cases}
$$

where $y_{1}, y_{2}, y_{3} \in \mathbb{C}$ are determined in (2.9) and $T_{0}$ are defined in Lemma 2.2. Applying the partial Fourier transform $\tilde{w}(t, \xi)=\mathscr{F}_{x \rightarrow \xi}(\tilde{u}(t, x))$ implies

$$
\begin{cases}\tilde{w}_{t}+\operatorname{diag}\left(y_{1}, y_{2}, y_{3}\right)|\xi|^{2} \tilde{w}=0, & t>0, \xi \in \mathbb{R}^{n}, \\ \tilde{w}(0, \xi)=T_{0}^{-1} w_{0}^{(0)}(\xi), & \xi \in \mathbb{R}^{n} .\end{cases}
$$

The solution to (5.31) is explicitly given by

$$
\tilde{w}(t, \xi)=\operatorname{diag}\left(e^{-y_{1}|\xi|^{2} t}, e^{-y_{2}|\xi|^{2} t}, e^{-y_{3}|\xi|^{2} t}\right) T_{0}^{-1} w_{0}^{(0)}(\xi) .
$$

Remark 5.5. From (5.30), the reference system is consisted of evolution equations

$$
\tilde{u}_{t}^{(j)}-\operatorname{Re} y_{j} \Delta \tilde{u}^{(j)}-i \operatorname{Im} y_{j} \Delta \tilde{u}^{(j)}=0,
$$

for $j=1,2,3$. Here we interpret this effect as classical diffusion phenomenon. Moreover, we have to point out that the reference system (5.30) is consisted of heat systems and Schrödinger systems due to the fact that Re $y_{j}>0$ and Im $y_{j} \neq 0$ for all $j=1,2,3$.

Remark 5.6. If one considers the reference system as the following heat system only:

$$
\tilde{u}_{t}-\operatorname{diag}\left(\operatorname{Re} y_{1}, \text { Re } y_{2} \text {, Re } y_{3}\right) \Delta \tilde{u}=0,
$$

or the following Schrödinger system only:

$$
\tilde{u}_{t}-i \operatorname{diag}\left(\operatorname{Im} y_{1}, \operatorname{Im} y_{2}, \operatorname{Im} y_{3}\right) \Delta \tilde{u}=0,
$$

we cannot observe any diffusion structure for $\sigma \in(1,2]$. In other words, comparing with Theorems 5.1 and 5.2, respectively, we observe that there is not any improvement in the decay estimates for the difference between the solutions to the system (2.4) with $\sigma \in(1,2]$ and the solutions to the reference systems (5.33) or (5.34).

Similar as last subsection, one can prove the following results.

Theorem 5.3. Let us consider the Cauchy problem (2.4) with $\sigma \in(1,2]$. We assume $\left(|D|^{2} u_{0}, u_{1}, \theta_{0}\right) \in$ $L^{m}\left(\mathbb{R}^{n}\right) \times L^{m}\left(\mathbb{R}^{n}\right) \times L^{m}\left(\mathbb{R}^{n}\right)$ with $m \in[1,2]$. Then, we have the following refinement estimates:

$$
\begin{aligned}
\| \chi_{\text {int }}(D) & \mathscr{F}_{\xi \rightarrow x}^{-1}\left(w^{(0)}-T_{0} \tilde{w}\right)(t, \cdot) \|_{\dot{H}^{s}\left(\mathbb{R}^{n}\right)} \\
& \lesssim(1+t)^{-\frac{(2-m) n+2 m s}{4 m}}-(\sigma-1)\left\|\left(|D|^{2} u_{0}, u_{1}, \theta_{0}\right)\right\|_{L^{m}\left(\mathbb{R}^{n}\right) \times L^{m}\left(\mathbb{R}^{n}\right) \times L^{m}\left(\mathbb{R}^{n}\right)},
\end{aligned}
$$

where $T_{0}$ are defined in Lemma 2.2.

Theorem 5.4. Let us consider the Cauchy problem (2.4) with $\sigma \in(1,2]$. We assume $\left(|D|^{2} u_{0}, u_{1}, \theta_{0}\right) \in$ $\delta\left(\mathbb{R}^{n}\right) \times \mathcal{S}\left(\mathbb{R}^{n}\right) \times \mathcal{S}\left(\mathbb{R}^{n}\right)$. Then, we have the next refinement estimates:

$$
\begin{aligned}
\| \chi_{\mathrm{int}}(D) & \mathscr{F}_{\xi \rightarrow x}^{-1}\left(w^{(0)}-T_{0} \tilde{w}\right)(t, \cdot) \|_{\dot{H}_{q}^{s}\left(\mathbb{R}^{n}\right)} \\
& \lesssim(1+t)^{-\frac{s}{2}-\frac{n}{2}\left(\frac{1}{p}-\frac{1}{q}\right)-(\sigma-1)}\left\|\left(|D|^{2} u_{0}, u_{1}, \theta_{0}\right)\right\|_{L^{p}\left(\mathbb{R}^{n}\right) \times L^{p}\left(\mathbb{R}^{n}\right) \times L^{p}\left(\mathbb{R}^{n}\right)},
\end{aligned}
$$

where $T_{0}$ are defined in Lemma 2.2 with $s \geq 0,1 \leq p \leq 2 \leq q \leq \infty$. 
Remark 5.7. From Theorems 5.1 5.2, 5.3 and 5.4, the diffusion structure appears for the Cauchy problem (1.2) with $\sigma \in[0,1) \cup(1,2]$. More precisely, comparing Theorems 4.2 and 4.4 with Theorems 5.1 and 5.2, respectively, we observe that the decay rate can be improved by $-\frac{1-\sigma}{2-\sigma}$ if $\sigma \in[0,1)$ as $t \rightarrow \infty$. In addition, comparing Theorems 4.2 and 4.4 with Theorems 5.3 and 5.4 , respectively, we observe that the decay rate can be improved by $-(\sigma-1)$ if $\sigma \in(1,2]$ as $t \rightarrow \infty$.

Remark 5.8. According to Theorems 5.3 and 5.4, the thermal dissipation generated by Fourier's law has a dominant influence in comparison with structural damping on diffusion phenomena when $\sigma \in(1,2]$.

\section{Asymptotic profiles of solutions}

Our main purpose in this section is to give asymptotic profiles of solutions to the Cauchy problem (1.2) in a framework of the weighted $L^{1}$ data. The idea is motivated by $[15,16]$.

In Section 4 we derived the following estimates for upper bounds of solutions with weighted $L^{1}$ data:

$$
\|U(t, \cdot)\|_{\dot{H}^{s}\left(\mathbb{R}^{n}\right)} \lesssim(1+t)^{-\gamma(\sigma, n, 1, s+1)}\left\|U_{0}\right\|_{H^{s}\left(\mathbb{R}^{n}\right) \cap L^{1,1}\left(\mathbb{R}^{n}\right)}+(1+t)^{-\gamma(\sigma, n, 1, s)}\left|\int_{\mathbb{R}^{n}} U_{0}(x) d x\right|,
$$

where $\sigma \in[0,2], n \geq 1, s \geq 0$. Here the solution $U(t, x)$ and data $U_{0}(x)$ are defined in $(2.5)$ and (2.6), respectively.

The natural questions are as follows. What is the estimate for the lower bounds of $\|U(t, \cdot)\|_{\dot{H}^{s}\left(\mathbb{R}^{n}\right)}$ in a framework of weighted $L^{1}$ data? Does this estimate is sharp? To answer these questions, we show some useful lemmas initially. Here Lemmas 6.1 and 6.2 have been proved in the papers [12, 14].

Lemma 6.1. Let $f \in L^{1}\left(\mathbb{R}^{n}\right)$. Then, we can expand $\hat{f}(\xi)$ by

$$
\hat{f}(\xi)=A_{f}(\xi)-i B_{f}(\xi)+P_{f} \text { for all } \xi \in \mathbb{R}^{n},
$$

where

$$
\begin{aligned}
A_{f}(\xi) & :=(2 \pi)^{-\frac{n}{2}} \int_{\mathbb{R}^{n}}(\cos (x \cdot \xi)-1) f(x) d x, \\
B_{f}(\xi) & :=(2 \pi)^{-\frac{n}{2}} \int_{\mathbb{R}^{n}} \sin (x \cdot \xi) f(x) d x, \\
P_{f} & :=(2 \pi)^{-\frac{n}{2}} \int_{\mathbb{R}^{n}} f(x) d x .
\end{aligned}
$$

Lemma 6.2. Let us consider $A_{f}(\xi)$ and $B_{f}(\xi)$ defined in Lemma 6.1. Then, we have the following estimates for them:

$$
\begin{aligned}
&\left|A_{f}(\xi)\right| \lesssim|\xi||| f \|_{L^{1,1}\left(\mathbb{R}^{n}\right)}, \\
&\left|B_{f}(\xi)\right| \lesssim|\xi||| f \|_{L^{1,1}\left(\mathbb{R}^{n}\right)} .
\end{aligned}
$$

Lemma 6.3. Let us consider $s \geq 0$ and $\alpha_{2}>0$. Then, the following estimate holds:

$$
\left\|\chi_{\mathrm{int}}(\xi)|\xi|^{s} e^{-c|\xi|^{\alpha_{2}} t}\right\|_{L^{2}\left(\mathbb{R}^{n}\right)} \gtrsim(1+t)^{-\frac{2 s+n}{2 \alpha_{2}}},
$$

where the constant $c>0$.

Proof. By directly calculation, we obtain

$$
\begin{aligned}
\left\|\chi_{\mathrm{int}}(\xi)|\xi|^{s} e^{-c|\xi|^{\alpha_{2}} t}\right\|_{L^{2}\left(\mathbb{R}^{n}\right)}^{2} & =\int_{\mathbb{R}^{n}} \chi_{\text {int }}^{2}(\xi)|\xi|^{2 s} e^{-2 c|\xi|^{\alpha_{2}} t} d \xi \\
& =\int_{0}^{\varepsilon} \int_{|\xi|=r} r^{2 s} e^{-2 c r^{\alpha_{2}} t} d S_{\xi} d r \\
& =\omega_{n} \int_{0}^{\varepsilon} r^{2 s+n-1} e^{-2 c r^{\alpha_{2}} t} d r
\end{aligned}
$$


where $\omega_{n}=\int_{|\omega|=1} d \omega=\frac{2 \pi \frac{n}{2}}{\Gamma\left(\frac{n}{2}\right)}$. By using the ansatz $r^{\alpha_{2}} t=\tau$, we complete the proof of the lemma.

Theorem 6.1. Let us assume $U_{0} \in H^{s}\left(\mathbb{R}^{n}\right) \cap L^{1,1}\left(\mathbb{R}^{n}\right)$ with $\left|P_{U_{0}}\right| \neq 0$, where $s \geq 0$. Then, the solution $U=U(t, x)$ to the Cauchy problem (1.2) with $\sigma \in[0,1)$ satisfies the following estimates for $t \gg 1$ :

$$
t^{-\frac{n+2 s}{4(2-\sigma)}}\left|P_{U_{0}}\right| \lesssim\|U(t, \cdot)\|_{\dot{H}^{s}\left(\mathbb{R}^{n}\right)} \lesssim t^{-\frac{n+2 s}{4(2-\sigma)}}\left\|U_{0}\right\|_{H^{s}\left(\mathbb{R}^{n}\right) \cap L^{1,1}\left(\mathbb{R}^{n}\right)} .
$$

Proof. To begin with, let us define

$$
J_{4}(t,|\xi|):=T_{0} T_{1} T_{1 \frac{1}{2}} \operatorname{diag}\left(e^{-|\xi|^{4-2 \sigma} t}, e^{-|\xi|^{2} t}, e^{-|\xi|^{2 \sigma} t}\right) T_{1 \frac{1}{2}}^{-1} T_{1}^{-1} T_{0}^{-1} .
$$

We use Lemmas 6.1, 6.2 and Theorem 5.1 to get

$$
\begin{aligned}
& \left\|\chi_{\text {int }}(D) \mathscr{F}_{\xi \rightarrow x}^{-1}\left(w^{(0)}\right)-\chi_{\mathrm{int}}(D) \mathscr{F}_{\xi \rightarrow x}^{-1}\left(J_{4}(t,|\xi|)\right) P_{U_{0}}\right\|_{\dot{H}^{s}\left(\mathbb{R}^{n}\right)} \\
& =\| \chi_{\mathrm{int}}(D) \mathscr{F}_{\xi \rightarrow x}^{-1}\left(w^{(0)}-T_{0} T_{1} T_{1 \frac{1}{2}} \tilde{w}\right) \\
& \quad+\chi_{\mathrm{int}}(D) \mathscr{F}_{\xi \rightarrow x}^{-1}\left(J_{4}(t,|\xi|)\left(A_{U_{0}}(\xi)-i B_{U_{0}}(\xi)\right)\right) \|_{\dot{H}^{s}\left(\mathbb{R}^{n}\right)} \\
& \lesssim(1+t)^{-\gamma(\sigma, n, 1, s)-\frac{1-\sigma}{2-\sigma}}\left\|\left(|D|^{2} u_{0}, u_{1}, \theta_{0}\right)\right\|_{L^{1}\left(\mathbb{R}^{n}\right) \times L^{1}\left(\mathbb{R}^{n}\right) \times L^{1}\left(\mathbb{R}^{n}\right)} \\
& \quad+\left\|\chi_{\mathrm{int}}(\xi)|\xi|^{s+1} e^{-|\xi|^{4-2 \sigma} t}\right\|_{L^{2}\left(\mathbb{R}^{n}\right)}\left\|\left(|D|^{2} u_{0}, u_{1}, \theta_{0}\right)\right\|_{L^{1,1}\left(\mathbb{R}^{n}\right) \times L^{1,1}\left(\mathbb{R}^{n}\right) \times L^{1,1}\left(\mathbb{R}^{n}\right)} \\
& \lesssim(1+t)^{-\gamma(\sigma, n, 1, s)-\frac{1-\sigma}{2-\sigma}}\left\|\left(|D|^{2} u_{0}, u_{1}, \theta_{0}\right)\right\|_{L^{1}\left(\mathbb{R}^{n}\right) \times L^{1}\left(\mathbb{R}^{n}\right) \times L^{1}\left(\mathbb{R}^{n}\right)} \\
& \quad+(1+t)^{-\gamma(\sigma, n, 1, s+1)}\left\|\left(|D|^{2} u_{0}, u_{1}, \theta_{0}\right)\right\|_{L^{1,1}\left(\mathbb{R}^{n}\right) \times L^{1,1}\left(\mathbb{R}^{n}\right) \times L^{1,1}\left(\mathbb{R}^{n}\right)} \cdot
\end{aligned}
$$

To get the lower bounds estimates, we apply triangle inequality to obtain

$$
\begin{aligned}
&\left\|\chi_{\text {int }}(D) \mathscr{F}_{\xi \rightarrow x}^{-1}\left(w^{(0)}\right)\right\|_{\dot{H}^{s}\left(\mathbb{R}^{n}\right)} \\
& \geq\left\|\chi_{\text {int }}(D) \mathscr{F}_{\xi \rightarrow x}^{-1}\left(J_{4}(t,|\xi|)\right) P_{U_{0}}\right\|_{\dot{H}^{s}\left(\mathbb{R}^{n}\right)} \\
& \quad-\left\|\chi_{\text {int }}(D) \mathscr{F}_{\xi \rightarrow x}^{-1}\left(w^{(0)}\right)-\chi_{\mathrm{int}}(D) \mathscr{F}_{\xi \rightarrow x}^{-1}\left(J_{4}(t,|\xi|)\right) P_{U_{0}}\right\|_{\dot{H}^{s}\left(\mathbb{R}^{n}\right)} \\
& \gtrsim\left\|\chi_{\mathrm{int}}(\xi)|\xi|^{s}\left(e^{-|\xi|^{4-2 \sigma} t}+e^{-|\xi|^{2} t}+e^{-|\xi|^{2 \sigma} t}\right)\right\|_{L^{2}\left(\mathbb{R}^{n}\right)}\left|P_{U_{0}}\right| \\
& \quad-(1+t)^{-\gamma(\sigma, n, 1, s)-\frac{1-\sigma}{2-\sigma}}\left\|\left(|D|^{2} u_{0}, u_{1}, \theta_{0}\right)\right\|_{L^{1}\left(\mathbb{R}^{n}\right) \times L^{1}\left(\mathbb{R}^{n}\right) \times L^{1}\left(\mathbb{R}^{n}\right)} \\
& \quad-(1+t)^{-\gamma(\sigma, n, 1, s+1)}\left\|\left(|D|^{2} u_{0}, u_{1}, \theta_{0}\right)\right\|_{L^{1,1}\left(\mathbb{R}^{n}\right) \times L^{1,1}\left(\mathbb{R}^{n}\right) \times L^{1,1}\left(\mathbb{R}^{n}\right)} .
\end{aligned}
$$

In conclusion, for $t \gg 1$ the following estimate holds:

$$
\left\|\chi_{\text {int }}(D) \mathscr{F}_{\xi \rightarrow x}^{-1}\left(w^{(0)}\right)\right\|_{\dot{H}^{s}\left(\mathbb{R}^{n}\right)} \gtrsim t^{-\gamma(\sigma, n, 1, s)}\left|P_{U_{0}}\right| .
$$

Combining with the upper bounds estimate for $t \gg 1$ such that

$$
\left\|\mathscr{F}_{\xi \rightarrow x}^{-1}\left(w^{(0)}\right)\right\|_{\dot{H}^{s}\left(\mathbb{R}^{n}\right)} \lesssim t^{-\gamma(\sigma, n, 1, s)}\left\|U_{0}\right\|_{H^{s}\left(\mathbb{R}^{n}\right) \cap L^{1,1}\left(\mathbb{R}^{n}\right)}
$$

and

$$
\left\|\chi_{\text {int }}(D) \mathscr{F}_{\xi \rightarrow x}^{-1}\left(w^{(0)}\right)\right\|_{\dot{H}^{s}\left(\mathbb{R}^{n}\right)} \lesssim\left\|\mathscr{F}_{\xi \rightarrow x}^{-1}\left(w^{(0)}\right)\right\|_{\dot{H}^{s}\left(\mathbb{R}^{n}\right)},
$$

we complete the proof.

Theorem 6.2. Let us assume $U_{0} \in H^{s}\left(\mathbb{R}^{n}\right) \cap L^{1,1}\left(\mathbb{R}^{n}\right)$ with $\left|P_{U_{0}}\right| \neq 0$, where $s \geq 0$. Then, the solution $U=U(t, x)$ to the Cauchy problem $(1.2)$ with $\sigma \in(1,2]$ satisfies the following estimates for $t \gg 1$ :

$$
t^{-\frac{n+2 s}{4}}\left|P_{U_{0}}\right| \lesssim\|U(t, \cdot)\|_{\dot{H}^{s}\left(\mathbb{R}^{n}\right)} \lesssim t^{-\frac{n+2 s}{4}}\left\|U_{0}\right\|_{H^{s}\left(\mathbb{R}^{n}\right) \cap L^{1,1}\left(\mathbb{R}^{n}\right)} .
$$


Proof. Following the proof of Theorem 6.1 one can complete this proof.

Finally, we derive asymptotic profiles of solutions to the Cauchy problem (1.2) for the case $\sigma=1$.

Theorem 6.3. Let us assume $U_{0} \in H^{s}\left(\mathbb{R}^{n}\right) \cap L^{1,1}\left(\mathbb{R}^{n}\right)$ with $\left|P_{U_{0}}\right| \neq 0$, where $s \geq 0$. Then, the solution $U=U(t, x)$ to the Cauchy problem (1.2) with $\sigma=1$ satisfies the following estimates for $t \gg 1$ :

$$
t^{-\frac{n+2 s}{4}}\left|P_{U_{0}}\right| \lesssim\|U(t, \cdot)\|_{\dot{H}^{s}\left(\mathbb{R}^{n}\right)} \lesssim t^{-\frac{n+2 s}{4}}\left\|U_{0}\right\|_{H^{s}\left(\mathbb{R}^{n}\right) \cap L^{1,1}\left(\mathbb{R}^{n}\right)} .
$$

Proof. Let us define

$$
J_{5}(t,|\xi|):=T_{1,0} \operatorname{diag}\left(e^{-y_{4}|\xi|^{2} t}, e^{-y_{5}|\xi|^{2} t}, e^{-y_{6}|\xi|^{2} t}\right) T_{1,0}^{-1}
$$

From Theorem 2.3, we know

$$
\begin{aligned}
\| \chi_{\text {int }}(D) & \mathscr{F}_{\xi \rightarrow x}^{-1}\left(w^{(0)}\right)-\chi_{\mathrm{int}}(D) \mathscr{F}_{\xi \rightarrow x}^{-1}\left(J_{5}(t,|\xi|)\right) P_{U_{0}} \|_{\dot{H}^{s}\left(\mathbb{R}^{n}\right)} \\
& =\left\|\chi_{\mathrm{int}}(D) \mathscr{F}_{\xi \rightarrow x}^{-1}\left(J_{5}(t,|\xi|)\left(A_{U_{0}}(\xi)-i B_{U_{0}}(\xi)\right)\right)\right\|_{\dot{H}^{s}\left(\mathbb{R}^{n}\right)} \\
& \lesssim(1+t)^{-\frac{n+2 s}{4}-\frac{1}{2}}\left\|U_{0}\right\|_{L^{1,1}\left(\mathbb{R}^{n}\right)},
\end{aligned}
$$

where $T_{0,1}, y_{4}, y_{5}, y_{6}$ are defined in Lemma 2.3.

Then, repeating the procedure of the proof of Theorem 6.1 we derive for $t \gg 1$

$$
t^{-\frac{n+2 s}{4}}\left|P_{U_{0}}\right| \lesssim\left\|\chi_{\mathrm{int}}(D) \mathscr{F}_{\xi \rightarrow x}^{-1}\left(w^{(0)}\right)\right\|_{\dot{H}^{s}\left(\mathbb{R}^{n}\right)} \lesssim\left\|\mathscr{F}_{\xi \rightarrow x}^{-1}\left(w^{(0)}\right)\right\|_{\dot{H}^{s}\left(\mathbb{R}^{n}\right)},
$$

and the proof of Theorem 6.3 is complete.

Remark 6.1. According to Theorems 6.1, 6.2 and 6.3, the thermal dissipation generated by Fourier's law has a dominant influence in comparison with structural damping on long-time asymptotic profiles of solutions when $\sigma \in[1,2]$.

\section{Concluding remarks}

Remark 7.1. In general, our method to derive sharp asymptotic profiles of solutions in the framework of $L^{1,1}$ can be probably applied to the Cauchy problem for other systems in elastic material including elastic waves with different damping mechanisms, thermoelastic systems, thermodiffusion systems.

In detail, for elastic waves with friction or structural damping [29, 3], elastic waves with KelvinVoigt damping [2], thermoelastic systems [18, 37, 40, 41, 31] and thermodiffusion systems [25], the authors applied diagonalization procedures or asymptotic expansions of eigenvalues/eigenprojections to derive representations of solutions. By these representations of solutions, one may obtain diffusion phenomena with weighted $L^{1}$ data. Then, one can follow the method in Section 6 to derive the sharp estimates for lower bounds and upper bounds of solutions in a framework of weighted $L^{1}$ data.

\subsection{Summary}

In the following we will collect results for the Cauchy problem for thermoelastic plate equations with friction or structural damping (1.2).

In the paper we first derive Gevrey smoothing of solutions (see Table 1 ) and $L^{2}$ well-posedness for the Cauchy problem (1.2) such that

$$
U \in \mathscr{C}\left([0, \infty), L^{2}\left(\mathbb{R}^{n}\right)\right) \text { if we assume } U_{0} \in L^{2}\left(\mathbb{R}^{n}\right) .
$$

Next, we obtain several decay estimates of solutions. On one hand, we derive the following energy estimates:

$$
\|U(t, \cdot)\|_{\dot{H}^{s}\left(\mathbb{R}^{n}\right)} \lesssim(1+t)^{-\frac{(2-m) n+2 m s}{2 m K}}\left\|U_{0}\right\|_{H^{s}\left(\mathbb{R}^{n}\right) \cap L^{m}\left(\mathbb{R}^{n}\right)},
$$


where $s \geq 0, m \in[1,2]$, and

$$
\|U(t, \cdot)\|_{\dot{H}^{s}\left(\mathbb{R}^{n}\right)} \lesssim(1+t)^{-\frac{n+2(s+\delta)}{2 K}}\left\|U_{0}\right\|_{H^{s}\left(\mathbb{R}^{n}\right) \cap L^{1, \delta}\left(\mathbb{R}^{n}\right)}+(1+t)^{-\frac{n+2 s}{2 K}}\left|P_{U_{0}}\right|,
$$

where $s \geq 0, \delta \in(0,1]$. Here some numbers $K$ (specified in the table below). On the other hand, there are $L^{p}-L^{q}$ estimates not necessary on the conjugate line of the form

$$
\|U(t, \cdot)\|_{\dot{H}_{q}^{s}\left(\mathbb{R}^{n}\right)} \lesssim(1+t)^{-\frac{s}{K}-\frac{n}{K}\left(\frac{1}{p}-\frac{1}{q}\right)}\left\|U_{0}\right\|,
$$

for suitable $p, q$ and some numbers $K$ (specified in the table below). Here $\left\|U_{0}\right\|$ corresponds to initial data measured in an appropriate norm, which is based on $L^{p}$.

Finally, we derive diffusion phenomena with data belonging to different function spaces, and asymptotic profiles of solutions with weighted $L^{1}$ data

$$
t^{-\frac{n+2 s}{2 K}}\left|P_{U_{0}}\right| \lesssim\|U(t, \cdot)\|_{\dot{H}^{s}\left(\mathbb{R}^{n}\right)} \lesssim t^{-\frac{n+2 s}{2 K}}\left\|U_{0}\right\|_{H^{s}\left(\mathbb{R}^{n}\right) \cap L^{1,1}\left(\mathbb{R}^{n}\right)}
$$

\begin{tabular}{|c|c|c|c|c|c|c|}
\hline & $\sigma=0$ & $\sigma \in(0,1)$ & $\sigma=1$ & $\sigma \in(1,3 / 2]$ & $\sigma \in(3 / 2,2)$ & $\sigma=2$ \\
\hline $\begin{array}{c}\text { Gevrey } \\
\text { smoothing }\end{array}$ & \multicolumn{4}{|c|}{$\Gamma^{1}\left(\mathbb{R}^{n}\right)$ (analytic smoothing) } & $\Gamma^{\frac{1}{4-2 \sigma}}\left(\mathbb{R}^{n}\right)$ & - \\
\hline $\begin{array}{c}\text { Energy } \\
\text { estimates }\end{array}$ & \multicolumn{2}{|c|}{$K=4-2 \sigma$} & \multicolumn{4}{|c|}{$K=2$} \\
\hline $\begin{array}{c}L^{p}-L^{q} \\
\text { estimates }\end{array}$ & \multicolumn{2}{|c|}{$\begin{array}{l}K=4-2 \sigma \text { and } \\
1 \leq p \leq 2 \leq q \leq \\
\infty\end{array}$} & $\begin{array}{c}K=2 \text { and } \\
1 \leq p \leq q \leq \infty\end{array}$ & \multicolumn{3}{|c|}{$\begin{array}{l}K=2 \text { and } \\
1 \leq p \leq 2 \leq q \leq \\
\infty\end{array}$} \\
\hline $\begin{array}{l}\text { Diffusion } \\
\text { phenomena } \\
\text { (dif. phe.) }\end{array}$ & $\begin{array}{l}\text { double } \\
\text { dif. phe. }\end{array}$ & $\begin{array}{l}\text { triple } \\
\text { dif. phe. }\end{array}$ & - & \multicolumn{3}{|c|}{ single dif. phe. } \\
\hline $\begin{array}{l}\text { Asymptotic } \\
\text { profiles }\end{array}$ & \multicolumn{2}{|c|}{$K=4-2 \sigma$} & & \multicolumn{3}{|c|}{$K=2$} \\
\hline
\end{tabular}

for $t \gg 1$, where $\left|P_{U_{0}}\right| \neq 0, s \geq 0$ and some numbers $K$ are chosen in Table 1 .

TABLE 1. Summary for qualitative properties of solutions

We should point out that when $K=4$, friction has a dominant influence in the corresponding decay estimates; when $K=4-2 \sigma$, structural damping has a dominant influence in the corresponding decay estimates; when $K=2$, thermal dissipation generated by Fourier's law has a dominant influence in the corresponding decay estimates.

\subsection{Estimates for the solution itself}

Throughout this paper, we apply diagonalization procedure to get the representations of solutions

$$
U(t, x)=\left(u_{t}+|D|^{2} u, u_{t}-|D|^{2} u, \theta\right)^{\mathrm{T}}(t, x)
$$

and study some qualitative properties of solutions to the Cauchy problem (1.2).

Nevertheless, up to now, concerning the qualitative properties of the solution $u=u(t, x)$ to the Cauchy problem (1.2), we did not derive any estimate for the solution itself. In this section, we will show some strategies to derive estimates for the solution itself. We propose three different strategies.

Strategy 1. Estimates of $u$ by using the Riesz potential theory.

We formally define the Riesz potential in $\mathbb{R}^{n}$ by its action on a measurable function $f=f(x)$ by convolution, that is

$$
\left(\tilde{I}_{2 \kappa} f\right)(x) \equiv\left(\tilde{I}_{2 \kappa} * f\right)(x):=\mathscr{F}^{-1}\left(|\xi|^{-2 \kappa} \hat{f}(\xi)\right)(x) \equiv C_{n, \kappa} \int_{\mathbb{R}^{n}} \frac{f(y)}{|x-y|^{n-2 \kappa}} d y
$$

where $\kappa \in\left(0, \frac{n}{2}\right)$.

The study of the following mapping properties to $\tilde{I}_{2 \kappa}$ was initiated by [35]. 
Lemma 7.1. Let us assume $f \in L^{p}\left(\mathbb{R}^{n}\right)$ for $p \in\left(1, \frac{n}{2 \kappa}\right)$. Then, $\tilde{I}_{2 \kappa} f \in L^{p^{*}}\left(\mathbb{R}^{n}\right)$, where

$$
\left\|\tilde{I}_{2 \kappa} f\right\|_{L^{p^{*}\left(\mathbb{R}^{n}\right)}} \lesssim\|f\|_{L^{p}\left(\mathbb{R}^{n}\right)} \text { with } \frac{1}{p}-\frac{1}{p^{*}}=\frac{2 \kappa}{n} .
$$

Then, we may estimates the solution itself by

$$
\begin{aligned}
\|u(t, \cdot)\|_{L^{q}\left(\mathbb{R}^{n}\right)} & \lesssim\left\|\mathscr{F}_{\xi \rightarrow x}^{-1}\left(|\xi|^{-2} w^{(0)}\right)(t, \cdot)\right\|_{L^{q}\left(\mathbb{R}^{n}\right)}=\left\|\tilde{I}_{2} \mathscr{F}_{\xi \rightarrow x}^{-1}\left(w^{(0)}\right)(t, \cdot)\right\|_{L^{q}\left(\mathbb{R}^{n}\right)} \\
& \lesssim\left\|\mathscr{F}_{\xi \rightarrow x}^{-1}\left(w^{(0)}\right)(t, \cdot)\right\|_{L^{\frac{2 q+n}{n q}\left(\mathbb{R}^{n}\right)}},
\end{aligned}
$$

where $0<\frac{1}{q}<1-\frac{2}{n}$. Next, following a similar procedure of Section 4 , one may complete estimates of the solution itself.

Strategy 2. Estimates of $u$ by using the integral formula.

For the case $\frac{1}{q} \in[0,1] \backslash\left(0,1-\frac{2}{n}\right)$, we cannot apply Strategy 1 . Therefore, by the integral formula

$$
u(t, x)-u(0, x)=\int_{0}^{t} u_{\tau}(\tau, x) d \tau
$$

we obtain

$$
\|u(t, \cdot)\|_{L^{q}\left(\mathbb{R}^{n}\right)} \lesssim\left\|u_{0}\right\|_{L^{q}\left(\mathbb{R}^{n}\right)}+\int_{0}^{t}\left\|u_{\tau}(\tau, \cdot)\right\|_{L^{q}\left(\mathbb{R}^{n}\right)} d \tau .
$$

Next, we apply the estimates of $u_{\tau}(\tau, \cdot)$ in the $L^{q}$ norm to complete estimates of the solution itself. We should remark that to apply this strategy, we need to take an additional assumption on the first data such that $u_{0} \in L^{q}\left(\mathbb{R}^{n}\right)$.

Strategy 3. Estimates of $u$ by using the representation of the solution.

By some direct calculations, we may transfer the Cauchy problem (1.2) to the following Cauchy problem for third-order equation:

$$
\begin{cases}u_{t t t}+(-\Delta)^{\sigma} u_{t t}-\Delta u_{t t}+2 \Delta^{2} u_{t}+(-\Delta)^{\sigma+1} u_{t}-\Delta^{3} u=0, & t>0, x \in \mathbb{R}^{n}, \\ \left(u, u_{t}, u_{t t}\right)(0, x)=\left(u_{0}, u_{1}, u_{2}\right)(x), & x \in \mathbb{R}^{n},\end{cases}
$$

where $\sigma \in[0,2]$ and

$$
u_{2}(x):=-\Delta^{2} u_{0}(x)-(-\Delta)^{\sigma} u_{1}(x)-\Delta \theta_{0}(x) .
$$

Applying the partial Fourier transformation with respect to spatial variables to (7.36), we obtain an ordinary differential equation depending on the parameter $|\xi|$

$$
\begin{cases}\hat{u}_{t t t}+\left(|\xi|^{2 \sigma}+|\xi|^{2}\right) \hat{u}_{t t}+\left(2|\xi|^{4}+|\xi|^{2 \sigma+2}\right) \hat{u}_{t}+|\xi|^{6} \hat{u}=0, & t>0, \xi \in \mathbb{R}^{n}, \\ \left(\hat{u}, \hat{u}_{t}, \hat{u}_{t t}\right)(0, \xi)=\left(\hat{u}_{0}, \hat{u}_{1}, \hat{u}_{2}\right)(\xi), & \xi \in \mathbb{R}^{n} .\end{cases}
$$

The characteristic roots $\lambda_{j}=\lambda_{j}(|\xi|), j=1,2,3$, for the equation of (7.37) satisfy the parameter dependent cubic equation

$$
\lambda^{3}+\left(|\xi|^{2 \sigma}+|\xi|^{2}\right) \lambda^{2}+\left(2|\xi|^{4}+|\xi|^{2 \sigma+2}\right) \lambda+|\xi|^{6}=0 .
$$

We may find the exact solution of (7.38) as follows:

$$
\lambda_{j}=r_{3, j}-\frac{r_{1}}{3 r_{3, j}}-\frac{|\xi|^{2 \sigma}+|\xi|^{2}}{3},
$$


where

$$
\begin{aligned}
& r_{1}=\frac{1}{3}\left(5|\xi|^{4}+|\xi|^{2 \sigma+2}-|\xi|^{4 \sigma}\right), \\
& r_{2}=\frac{1}{27}\left(11|\xi|^{6}+2|\xi|^{6 \sigma}-3|\xi|^{4 \sigma+2}-2|\xi|^{2 \sigma+4}\right), \\
& r_{3}^{3}=\frac{1}{2}\left(-r_{2} \pm \sqrt{r_{2}^{2}+\frac{4}{27} r_{1}^{3}}\right), \text { where } r_{3,1}, r_{3,1}, r_{3,1} \text { are complex solutions of it. }
\end{aligned}
$$

It provides an opportunity for us to derive explicit representations of solutions to (7.37) such that

$$
\hat{u}(t, \xi)=c_{1}(\xi) e^{\lambda_{1}(|\xi|) t}+c_{2}(\xi) e^{\lambda_{2}(|\xi|) t}+c_{3}(\xi) e^{\lambda_{3}(|\xi|) t},
$$

where $\lambda_{j}(|\xi|)$ are given by (7.39) and the coefficients $c_{j}(\xi)$ are given by

$$
\begin{aligned}
& c_{1}(\xi)=\frac{\lambda_{2}(|\xi|) \lambda_{3}(|\xi|) \hat{u}_{0}(\xi)-\lambda_{2}(|\xi|) \hat{u}_{1}(\xi)-\lambda_{3}(|\xi|) \hat{u}_{1}(\xi)-|\xi|^{4} \hat{u}_{0}(\xi)-|\xi|^{2 \sigma} \hat{u}_{1}(\xi)+|\xi|^{2} \hat{\theta}_{0}(\xi)}{\lambda_{1}^{2}(|\xi|)-\lambda_{1}(|\xi|) \lambda_{2}(|\xi|)-\lambda_{1}(|\xi|) \lambda_{3}(|\xi|)+\lambda_{2}(|\xi|) \lambda_{3}(|\xi|)} \\
& c_{2}(\xi)=\frac{\lambda_{1}(|\xi|) \lambda_{3}(|\xi|) \hat{u}_{0}(\xi)-\lambda_{1}(|\xi|) \hat{u}_{1}(\xi)-\lambda_{3}(|\xi|) \hat{u}_{1}(\xi)-|\xi|^{4} \hat{u}_{0}(\xi)-|\xi|^{2 \sigma} \hat{u}_{1}(\xi)+|\xi|^{2} \hat{\theta}_{0}(\xi)}{\lambda_{2}^{2}(|\xi|)-\lambda_{1}(|\xi|) \lambda_{2}(|\xi|)+\lambda_{1}(|\xi|) \lambda_{3}(|\xi|)-\lambda_{2}(|\xi|) \lambda_{3}(|\xi|)} \\
& c_{3}(\xi)=\frac{\lambda_{1}(|\xi|) \lambda_{2}(|\xi|) \hat{u}_{0}(\xi)-\lambda_{1}(|\xi|) \hat{u}_{1}(\xi)-\lambda_{2}(|\xi|) \hat{u}_{1}(\xi)-|\xi|^{4} \hat{u}_{0}(\xi)-|\xi|^{2 \sigma} \hat{u}_{1}(\xi)+|\xi|^{2} \hat{\theta}_{0}(\xi)}{\lambda_{3}^{2}(|\xi|)+\lambda_{1}(|\xi|) \lambda_{2}(|\xi|)-\lambda_{1}(|\xi|) \lambda_{3}(|\xi|)-\lambda_{2}(|\xi|) \lambda_{3}(|\xi|)} .
\end{aligned}
$$

It is possible to derive estimates for $u=u(t, x)$ by applying these representations.

\section{Acknowledgments}

The PhD study of Mr. Wenhui Chen is supported by Sächsiches Landesgraduiertenstipendium. The author thank his supervisor Michael Reissig for the suggestions in the preparation of the final version.

\section{References}

[1] G. Avalos and I. Lasiecka, Exponential stability of a thermoelastic system without mechanical dissipation, Rend. Istit. Mat. Univ. Trieste Suppl., 28:1-28, 1997.

[2] W. Chen, Decay properties and asymptotic profiles for elastic waves with Kelvin-Voigt damping in 2D, Preprint, 2019. arXiv:1811.02223

[3] W. Chen and M. Reissig, Weakly coupled systems of semilinear elastic waves with different damping mechanisms in 3D, Math. Methods Appl. Sci., 42(2):667-709, 2019.

[4] M. D'Abbicco and M.R. Ebert, Diffusion phenomena for the wave equation with structural damping in the $L^{p}-L^{q}$ framework, J. Differential Equations, 256(7):2307-2336, 2014.

[5] R. Denk and R. Racke, $L^{p}$-resolvent estimates and time decay for generalized thermoelastic plate equations, Electron. J. Differential Equations, 48:16 pp, 2006.

[6] R. Denk, R. Racke, and Y. Shibata, $L_{p}$ theory for the linear thermoelastic plate equations in bounded and exterior domains, Adv. Differential Equations, 14(7-8):685-715, 2009.

[7] R. Denk, R. Racke, and Y. Shibata, Local energy decay estimate of solutions to the thermoelastic plate equations in two- and three-dimensional exterior domains, Z. Anal. Anwend, 29(1):21-62, 2010.

[8] M.R. Ebert and M. Reissig, Methods for partial differential equations. Qualitative properties of solutions, phase space analysis, semilinear models, Birkhäuser, 2018.

[9] L.H. Fatori, M.A. Jorge Silva, T.F. Ma, and Z. Yang, Long-time behavior of a class of thermoelastic plates with nonlinear strain, J. Differential Equations, 259(9):4831-4862, 2015.

[10] L. Fischer and R. Racke, Ill-posedness of coupled systems with delay, London Math. Soc. Lecture Note Ser., 439:151-160, 2018.

[11] H. Wu, Long-time behavior for a nonlinear plate equation with thermal memory, J. Math. Anal. Appl., 348(2):650-670, 2008. 
[12] J.L. Horbach, R. Ikehata, and R.C. Charão, Optimal decay rates and asymptotic profile for the plate equation with structural damping, J. Math. Anal. Appl., 440(2):529-560, 2016.

[13] K. Ide, K. Haramoto, and S. Kawashima, Decay property of regularity-loss type for dissipative Timoshenko system, Math. Models Methods Appl. Sci., 18(5):647-667, 2008.

[14] R. Ikehata, New decay estimates for linear damped wave equations and its application to nonlinear problem, Math. Methods Appl. Sci., 27(8):865-889, 2004.

[15] R. Ikehata, Revisit on how to derive asymptotic profiles to some evolution equations, Preprint, 2013. arXiv:1506.04858

[16] R. Ikehata, Asymptotic profiles for wave equations with strong damping, J. Differential Equations, 257(6):2159-2177, 2014.

[17] R. Ikehata and M. Soga, Asymptotic profiles for a strongly damped plate equation with lower order perturbation, Commun. Pure Appl. Anal., 14(5):1759-1780, 2015.

[18] K. Jachmann, A unified treatment of models of thermoelasticity, PhD Thesis, TU Bergakademie Freiberg, 2008.

[19] J.U. Kim, On the energy decay of a linear thermoelastic bar and plate, SIAM J. Math. Anal., 23(4):889-899, 1992.

[20] I. Lasiecka and R. Triggiani, Two direct proofs on the analyticity of the s.c. semigroup arising in abstract thermo-elastic equations, Adv. Differential Equations, 3(3):387-416, 1998.

[21] I. Lasiecka and R. Triggiani, Analyticity, and lack thereof, of thermo-elastic semigroups, ESAIM, Proc., 4:199-222, 1998.

[22] I. Lasiecka and R. Triggiani, Analyticity of thermo-elastic semigroups with coupled hinged/Neumann B.C., Abstr. Appl. Anal., 3(1-2):153-169, 1998.

[23] I. Lasiecka and R. Triggiani, Analyticity of thermo-elastic semigroups with free boundary conditions, Ann. Scuola Norm. Sup. Pisa CI. Sci. (4), 27(3-4):457-482, 1998.

[24] K. Liu and Z. Liu, Exponential stability and analyticity of abstract linear thermoelastic systems, Z. Angew. Math. Phys., 48(6):885-904, 1997.

[25] Y. Liu and M. Reissig, Models of thermodiffusion in 1D, Math. Methods Appl. Sci., 37(6):817-837, 2014.

[26] J.E. Muñoz Rivera and R. Racke, Smoothing properties, decay, and global existence of solutions to nonlinear coupled systems of thermoelastic type, SIAM J. Math. Anal., 26(6):1547-1563, 1995.

[27] J.E. Muñoz Rivera and R. Racke, Large solutions and smoothing properties for nonlinear thermoelastic systems, J. Differential. Equations, 127(2):454-483, 1996.

[28] D.T. Pham, M. Mohamed Kainane, and M. Reissig, Global existence for semi-linear structurally damped $\sigma$-evolution models, J. Math. Anal. Appl., 431(1):569-596, 2015.

[29] M. Reissig, Structurally damped elastic waves in 2D, Math. Methods Appl. Sci., 39(15):4618-4628, 2016.

[30] M. Reissig and J. Smith, $L^{p}-L^{q}$ estimate for wave equation with bounded time dependent coefficient, Hokkaido Math. J., 34(3):541-586, 2005.

[31] M. Reissig and Y.G. Wang, Cauchy problems for linear thermoelastic systems of type III in one space variable, Math. Methods Appl. Sci., 28(11):1359-1381, 2005.

[32] R. Racke and Y. Ueda, Dissipative structures for thermoelastic plate equations in $\mathbb{R}^{n}$, Adv. Differential Equations, 21(7-8):610-630, 2016.

[33] L. Radino, Linear partial differential operators in Gevrey spaces, World Scientific Publishing Co., Inc., River Edge, NJ, 1993.

[34] B. Said-Houari, Decay properties of linear thermoelastic plates: Cattaneo versus Fourier law, Appl. Anal., 92(2):424-440, 2013.

[35] S.L. Sobolev, On a theorem of functional analysis, Mat. Sbornik, 4(4):471-497, 1938.

[36] D. Wang and Y. Wang, Pullback attractor for $N$-dimensional thermoelastic coupled structure equations, Bound. Value Probl., 5:21 pp, 2018.

[37] Y.G. Wang and L. Yang, $L^{p}-L^{q}$ decay estimates for Cauchy problems of linear thermoelastic systems with second sound in three dimensions, Proc. Roy. Soc. Edinburgh Sect. A, 136(1):189-207, 2006.

[38] D. Wang and J. Zhang, Long-time dynamics of $N$-dimensional structure equations with thermal memory, Bound. Value Probl., 136:21 pp, 2017. 
[39] K. Yagdjian, The Cauchy problem for hyperbolic operators. Multiple characteristics. Micro-local approach, Mathematical Topics, Akademie Verlag, Berlin, 1997.

[40] L. Yang and Y.G. Wang, $L^{p}-L^{q}$ decay estimates for the Cauchy problem of linear thermoelastic systems with second sound in one space variable, Quart. Appl. Math., 64(1):1-15, 2006.

[41] L. Yang and Y.G. Wang, Well-posedness and decay estimates for Cauchy problems of linear thermoelastic systems of type III in 3-D, Indiana Univ. Math. J., 55(4):1333-1361, 2006.

Wenhui Chen

Institute of Applied Analysis, Faculty for Mathematics and Computer Science

Technical University Bergakademie Freiberg

Prüferstraße 9

09596 Freiberg

Germany

e-mail: Wenhui.Chen@student.tu-freiberg.de 